Christian Fleck

\title{
Der Impact Faktor-Fetischismus
}

\author{
»Wenn eine Kennzahl angegriffen wird, ist sie kein \\ guter Maßstab mehr." \\ Charles Goodhart ${ }^{1}$
}

Nahezu jede soziale Gruppe hat ihre eigenen Kennzahlen entwickelt, die für Zwecke des Vergleichs, der Bewertung und der Reihung benutzt werden. Sportsfreunde berechnen Erfolgsraten, Börsianer treffen Entscheidungen auf der Basis des KursGewinn-Verhältnisses, und gewöhnlichen Menschen wird geraten, ihr Wohlbefinden zu beobachten: Body-Mass-Index, Intelligenzquotient und ähnliche Zahlen informieren sie darüber, ob und wie sehr sie vom Durchschnitt abweichen. In ähnlicher Weise informieren uns einfach zu merkende Zahlen über das Wohlbefinden ganzer Gesellschaften: Wirtschaftswachstum, Exportquoten, Arbeitslosen- und Scheidungsrate und anderes mehr. Die akademische Welt bildet hier keine Ausnahme, ihr Gold-Standard ist mittlerweile der Impact Faktor.

Die meisten dieser Indikatoren durchliefen eine sehr ähnliche Entwicklung. In den frühen Jahren genügten einfache Zählungen (der Bevölkerung, der Steuerzahler und der Wehrpflichtigen), doch ziemlich bald setzten sich Verhältniszahlen durch. Diese folgen selten denselben Berechnungsroutinen. Die Scheidungsrate ignoriert alle Trennungen vordem nicht formell Verheirateter, die mit dem Lob einer hohen Exportquote implizit verbundene Aufforderung zur Nachahmung durch andere Nationen ignoriert deren Unmöglichkeit, die Arbeitslosenrate lässt all jene unberücksichtigt, die sich vom formellen Arbeitsmarkt zurückgezogen haben, und der mittlerweile zum selbstverständlichen Bestandteil gehobener Gesprächskultur gewordene Einwand, das Bruttosozialprodukt berücksichtige den Beitrag des Schwarzmarktes nicht, ist rascher zur Hand als ein auch nur rudimentäres Wissen um die Grundlagen der volkswirtschaftlichen Gesamtrechnung. Sehr häufig tragen einmal etablierte Kennzahlen späteren Entwicklungen nicht Rechnung, sondern werden in der ursprünglichen Form tradiert, während ihre Aussagekraft schwindet. In manchen Fällen änderte selbst einhellige Zurückweisung durch Experten nichts daran. ${ }^{2}$ Ähnliches geschah mit dem Impact Faktor, der Kennzahl, die die Bedeutung einer Zeitschrift zu erfassen behauptet. Er wird verwendet oder zumindest hingenommen, obwohl Kritiker auf seine Mängel verwiesen haben - aber als soziale Technologie hat er sich fest etabliert.

Die These, die hier vertreten wird, lautet: Die breite, wenn auch häufig widerwillige Akzeptanz des Impact Faktors (zuerst in den Natur-, mittlerweile aber auch

1 Übersetzung der englischen Zitate in diesem Text von C.F.

2 Fleck 2010.

Leviathan, 41. Jg., 4/2013, S. $611-646$ 
bereits in den Sozialwissenschaften) beruht darauf, dass er in der Lage zu sein scheint, (Rang-)Differenzen zwischen wissenschaftlichen Zeitschriften (und Autoren) abzubilden, die mit dem Rahmenwissen der Rezipienten kompatibel sind. Ein Blick auf die (sozialwissenschaftlichen) Zeitschriften mit dem höchsten Impact Faktor scheint die angesehensten richtig identifiziert zu haben, woraus aber (wie zu zeigen sein wird) unzulässigerweise geschlossen wird, der Impact Faktor sei auch in der Lage, die Abstände zu den und zwischen den weiter hinten platzierten Zeitschriften zutreffend erfasst zu haben. Eine öffentlich vorgetragene Kritik am Impact Faktor-Regime müsste daher von Etablierten vorgebracht werden, um Aussicht auf Erfolg zu haben. Die dem Modell legitimer Herrschaft gleichende Hinnahme des Impact Faktors durch diejenigen, die ihren akademischen Aufstieg noch vor sich haben, resultiert zum einen aus dem weit verbreiteten Nichtwissen nicht nur über die Details seiner Berechnung, sondern auch über die grundlegenden Entscheidungen, auf denen seine Kalkulation beruht. Andererseits scheint die (mutmaßlich) höhere Validität dieses Maßes für bestimmte Teile der Naturwissenschaften angesichts des größeren Ansehens, das diese Disziplinen in der breiteren Öffentlichkeit und insbesondere unter Wissenschaftsadministratoren und -politikern genießen, Nicht-Naturwissenschaftler zögern zu lassen, (wieder einmal) auf die Besonderheiten ihrer Kultur hinzuweisen. In der Spezialistenliteratur der Bibliometrie und Szientometrie herrscht hingegen weitgehend Konsens darüber, dass der Impact Faktor gravierende Mängel aufweist, weswegen dort eine große Zahl von alternativen Maßen vorgeschlagen wurde, die sich aber bislang gegen den Sieger Impact Faktor nicht durchzusetzen vermochten. Das Impact Faktor-Regime, das mittlerweile praktisch alle Wissenschaftsdisziplinen erfasst hat, wurde insbesondere in der Wissenschaftspolitik und -administration willkommen geheißen. Seine Legitimität resultiert wohl aus dem Zusammenwirken einer Wissensfragmentierung mit einer Prestigeordnung der wissenschaftlichen Disziplinen entlang ihrer Drittmittelaufbringung (beziehungsweise der mit Drittmittelschwäche gepaarten Unterlegenheitshaltung der Nicht-Naturwissenschaftler). Bemerkenswert ist daher, dass die jüngste internationale Protestaktion gegen die Verwendung des Impact Faktors von Medizinern und Naturwissenschaftlern initiiert wurde, während sich Namen von Sozialwissenschaftler nicht unter den Initianten finden. ${ }^{3}$ Der Impact Faktor-Fetischismus kann - wie jener, den Karl Marx meinte entdeckt zu haben - nur im Wege einer genauen Rekonstruktion seines Entstehens und gegenwärtigen Wirkens delegitimiert werden. Diese Aufklärungsarbeit soll im Folgenden versucht werden.

Am Beginn steht eine exemplarische Schilderung der gegenwärtigen Verwendung des Impact Faktors. Ich behandle dann seine Entstehung und zeige, dass anfangs kaum vermeidbare Festlegungen eine Pfadabhängigkeit eingeleitet haben. Das Ergebnis des Experimentierens von Bibliometrikern mit den ihnen zur Verfügung stehenden Daten einer sehr kleinen und speziellen Gruppe von Zeitschriften wurde

3 »The San Francisco Declaration on Research Assessment (DORA), initiated by the American Society for Cell Biology (ASCB) together with a group of editors and publishers of scholarly journals, recognizes the need to improve the ways in which the outputs of scientific research are evaluated. « (http://am.ascb.org/dora/; Zugriff vom 14.06.2013) 
umgehend generalisiert, ohne dass die Angemessenheit der Berechnungsmethode für die weiteren Fälle überprüft worden wäre. Danach untersuche ich die gegenwärtige Verwendung des Impact Faktors und mache am Beispiel europäischer soziologischer Zeitschriften auf fragwürdige Aspekte aufmerksam. Bedeutsame Entscheidungen wurden schlecht begründet, weshalb Verkürzungen und Irrtümer festzustellen sind. Das Ergebnis dieser detaillierten Untersuchung ist für diejenigen niederschmetternd, die die universelle Verwendbarkeit des Impact Faktors behaupten, ruinös für jene, die mit dem Impact Faktor Geld verdienen, und problematisch für all jene, die diese Kennzahlen für welche Zwecke auch immer verwenden. Abschließend zeige ich einige Gründe auf, warum der Impact Faktor in einigen Fächern - bei weitem nicht nur in der Soziologie - zum Gold-Standard wurde. ${ }^{4}$

\section{Gebrauch und Missbrauch des Impact Faktors}

Der Impact Faktor wird immer häufiger benutzt: Im Rahmen der britischen Forschungsevaluation beruhten die Ergebnisse des Research Assessment Exercise (RAE) (der alle fünf Jahre stattfindenden Verteilung von »Sternen " an Universitätsinstitute, deren Zahl künftige Mittelzuweisungen bestimmt) zumindest realiter auch auf bibliometrischen Daten, das European Research Council (das mit Mitteln der Europäischen Union Grundlagenforschung fördert) fordert von Bewerbern in der Schiene der Förderung von etablierten Forschern (advanced grants) die Angabe von Zitationszahlen, ${ }^{5}$ und zunehmend nutzen Universitäten Zitationsindizes, um Bewerber im Rahmen von Stellenbesetzungen und Beförderungen rascher selektieren zu können; asiatische Universitäten begannen damit, ihre Wissenschaftler nach der Zahl der in »Elite«-Zeitschriften veröffentlichten Aufsätze zu entlohnen. ${ }^{6}$ Das viel beachtete World University Ranking von Times Higher Education beruht zu einem wichtigen Teil auf Daten des » Web of Science« (WoS), was angesichts der Eigentumsverhältnisse wenig überraschend ist, wird diese Erhebung doch mittlerweile von eben jener Firma administriert - » powered by Thomson Reuters « $^{7}$-, die auch Eigentümer des WoS ist. Wenn es (Gruppen von) Wissenschaftlern im Zusammenhang mit geplanten Evaluationen gelingt, sich gegen Wünsche von Wissenschaftsverwaltern durchzusetzen, wird oft auf die Verwendung von Maßzahlen wie dem Impact Faktor ver-

4 Ich behaupte nicht, dass die Soziologie in irgendeiner Weise eine Besonderheit darstellt; vielmehr nehme ich an, dass das, was hier für diese Disziplin gezeigt wird, auch für viele andere zutreffen dürfte. Der Grund, warum ich mich auf die Soziologie beschränke, ist ein zweifacher: Zum einen ist es meine eigene Disziplin, und zum anderen sind Soziologen darin ausgebildet, Daten eine entsprechende kritische Würdigung angedeihen zu lassen und wenn das nicht einmal sie tun, dann wird man diese Haltung umso weniger bei anderen erwarten dürfen.

5 European Commission 2011, S. 26.

6 Fuyuno, Cyranoski 2006.

7 » The essential elements in our world-leading formula «; www.timeshighereducation.co.uk/ world-university-rankings/2012-13/world-ranking/methodology

(Zugriff vom 14.06.2013).

Leviathan, 41. Jg., 4/2013 
zichtet - so geschehen bei der Pilotstudie Forschungsrating Soziologie des deutschen Wissenschaftsrats und im Rahmen des britischen Research Excellence Framework, dem Nachfolger des eben erwähnten RAE, wo von den Nicht-Naturwissenschaften nur Psychologie und Ökonomie der Verwendung von Zitationsdaten zustimmten. Der sichtbaren Ablehnung und öffentlichen Kritik an Wissenschafts-Maßzahlen durch Professionsverbände steht allerdings die faktische Verwendung dieser Kennzahlen bei vielen Verfahren der Personalauswahl entgegen, mit paradoxem Ergebnis: Während einzelne (jüngere) Wissenschaftler sich mit einer für die Bewertung von Individuen prinzipiell ungeeigneten Vermessung ihrer Qualität konfrontiert sehen, gelingt es den akademischen Zünften (Professionsverbänden) und ihren Organisations- beziehungsweise Forschungseinheiten (Institute, Lehrstühle) noch, sich einer für sie im Vergleich eher angemessenen Bewertung zu entziehen. ${ }^{8}$

Die überwiegende Mehrzahl der internationalen soziologischen Zeitschriften nutzt den Impact Faktor als Ausweis ihrer Qualität. Alle Zeitschriften, die im Journal Citation Reports (JCR) 2011 die Ränge 1 bis 53 einnehmen, platzieren den aktuellen Impact Faktor prominent auf ihren Internetseiten, sei es allein oder gemeinsam mit anderen Angaben aus derselben Quelle, zum Beispiel dem Rang innerhalb wissenschaftlicher Disziplinen (sogenannter »subject categories «). In einigen wenigen Fällen findet man dort auch die Impact Faktoren vergangener Jahre angeführt, faktisch nie werden der Impact Faktor und seine Berechnung erläutert, geschweige denn relativiert. ${ }^{9}$ Ich fand nur 31 Zeitschriften, die darauf verzichteten,

8 Sehr häufig neigen Kritiker unter Hinweis darauf, dass intellektuelle Leistungen nicht quantifizierbar wären, dazu, auch die Basisannahmen hinter der Szientometrie und dem Impact Faktor abzulehnen. Stellvertretend für andere: Münch 2007; Kieser 2010; Collini 2012. Ausführlich: Fischer 2008. Im vorliegenden Aufsatz wird die Möglichkeit der Szientometrie nicht diskutiert, sondern nur die Berechnungs- und Verwendungsweisen des Impact Faktors untersucht. In Bezug auf mögliche Meriten einer quantitativen Analyse von Unterschieden zwischen Wissenschaftlern und ihren wissenschaftlichen Produkten (Texten) verhält sich der Autor agnostisch.

9 Einige Zeitschriften führen andere Informationen an, zum Beispiel: »European Sociological Review has received an increased impact factor of 1.935 , placing it in the top $10 \%$ in the field of Sociology « (http://esr.oxfordjournals.org/; Zugriff vom 14.06.2013); »2010 Journal Citation Reports ${ }^{\circledR}$ ranks Journal of Mathematical Sociology in the Mathematical Methods (social science), Sociology, and the Mathematics, Interdisciplinary Applications (science) categories « (www.tandfonline.com/action/aboutThisJournal?show=aimsScope \&journalCode=gmas20; Zugriff vom 14.06.2013); "News: The Economic and Social Review has been accepted for the Social Sciences Citation Index. More details to follow « (www.esr.ie/ESRPage2.html; Zugriff vom 14.06.2013); »A bibliometric study carried out by the Scientific Department `Social and Human Sciences` of the National Center for Scientific Research, Paris, published in his Bulletin, $\mathrm{n}^{\circ}$ 69, May 2004, ranked the Revue Française de Sociologie, as the leading French Journal with an international audience (www.rfs-revue.com/spip.php\%3Frubrique8\&lang=en.html; Zugriff vom 14.06.2013). 
ihren Impact Faktor publik zu machen - wenig überraschend waren diese Zeitschriften im Ranking weit hinten zu finden. ${ }^{10}$

Die University of Chicago Press verkündet auf ihrer Internetseite, dass ihre »Zeitschriften weiterhin hohe Impact-Faktoren erzielen «, listet dann elf ihrer Zeitschriften mit deren Rang in bestimmten Feldern einzeln auf und ergänzt diese Nachricht durch eine erklärende Notiz über den Impact Faktor:

»Journal Citation Reports ${ }^{\circledR}$ bietet dank quantifizierbarer statistischer auf Zitationsdaten beruhender Informationen ein systematisches, objektives Mittel um die führenden Zeitschriften der Welt kritisch zu bewerten. Indem JCR ${ }^{\circledR}$ die zitierten Referenzen zusammenträgt, hilft es Forschungseinflüsse und Bedeutung auf der Ebene der Zeitschrift und der Wissenschaftsdisziplin zu messen und zeigt die Beziehung zwischen zitierenden und zitierten Zeitschriften ${ }^{11}$

Kann man die Verwendung des Impact Faktors im Rahmen von Verlagspräsentationen noch als Werbeaktivität verstehen, erscheinen Bekundungen von Zeitschriftenherausgebern in einem etwas anderen Licht. Manche fühlen sich bemüßigt, »ihren «Impact Faktor hinauszuposaunen, um voller Stolz zu zeigen, wie sie gelobt werden. Die beiden Herausgeber der Ethnic and Racial Studies, Martin Bulmer und John Solomos, veröffentlichten im letzten Heft des Jahrgangs 2010 ein Editorial, in dem es unter anderem heißt: »Der Einfluss (impact) dieser Zeitschrift auf die breitere wissenschaftliche Gemeinschaft zeigt sich im verbesserten Impact Faktor, den wir 2010 vom ISI Social Science Citation Index für 2009 verliehen bekommen haben « (Bulmer, Solomos 2010, Hervorhebung C.F.).

Der Herausgeber der Besprechungszeitschrift Contemporary Sociology, Alan Sica, bezog sich jüngst in einer seiner Editor's Remarks auf »ungeplante Folgen « des Impact Faktors, den er für den »rückläufigen Status von kritischen Rezensionen in den letzten Jahrzehnten « verantwortlich macht. Im Anschluss berichtet er über Maßnahmen, die die Mitglieder des Editorial Board von Contemporary Sociology vorgeschlagen hätten, um in der näheren Zukunft den Impact Faktor zu verbessern. ${ }^{12}$ Die scheidenden Herausgeber Wolfgang Streeck und Jürgen Feick gingen noch einen Schritt weiter und informierten ihre Leser über Folgendes: " 2012 erhielt die Socio-Economic Review ihren ersten Impact-Faktor: Wir sind stolz, das unsere Zeitschrift eine starke 1.78 erreichte «. ${ }^{13}$

Hingegen baten die Herausgeber von mehr als 50 wissenschaftsgeschichtlichen Zeitschriften bei einem vergleichbaren Ranking kollektiv darum, nicht im European Reference Index for the Humanities (ERIH) berücksichtigt zu werden, da sie dessen Kriterien prinzipiell ablehnten. ${ }^{14}$

10 Die vier deutschsprachigen soziologischen Zeitschriften, die im SSCI dokumentiert werden, verweisen auf ihren Internetseiten allesamt nicht auf den Impact Faktor. In informeller Kommunikation wird er aber mittlerweile recht aufmerksam verfolgt.

11 www.press.uchicago.edu/pressReleases/2012/July/UCP_1207_ImpactFactor.html; Zugriff vom 14.06.2013.

12 Sica 2012, S. 138.

13 Streeck, Feick 2012.

14 Fox et al. 2009.

Leviathan, 41. Jg., 4/2013 
Wissenschaftler, die die Benutzung des Impact Faktors kommentieren, bezeichnen dessen Verwendung häufig mit Floskeln wie »Herausputzen « (window dressing), "Mitmachen beim Spiel « (gaming the game) oder verweisen auf Mythen oder Zeremonien. ${ }^{15}$ Derartige Redewendungen wird man kaum als hinreichend detaillierte Kritik qualifizieren können. Die meisten Autoren unterlassen es, den Umstand eingehender zu würdigen, dass eine fortgesetzte Benutzung eines anfangs umstrittenen Maßstabs selbstverstärkende Wirkungen zeitigt und das herkömmliche akademische Eindrucksmanagement verändern wird. ${ }^{16}$

Die boomende Zitations-Index- und Impact Faktor-Rhetorik beeinflusst das Verhalten gewöhnlicher Wissenschaftler in ambivalenter Form: Sehr wahrscheinlich wurde durch die Verlautbarung von Rangordnungen das Leseverhalten selbst modifiziert. ${ }^{17}$ Jeglicher Versuch, halbwegs informiert zu bleiben, erfordert eine Entscheidung darüber, welche Zeitschriften man einigermaßen regelmäßig durchblättert oder konsultiert, um für die eigene Forschung oder Lehre auf der Höhe der Zeit zu bleiben. In der Folge tauchen Artikel, die in Zeitschriften mit höherem Impact Faktor erschienen, rascher auf Literaturlisten auf als Aufsätze aus Zeitschriften von angeblich geringerer Bedeutung. ${ }^{18}$ Auf der anderen Seite versuchen wir alle - ganz unabhängig davon, dass wir zur gleichen Zeit über die steigende Bedeutung dessen klagen, was als " audit culture « bezeichnet wurde ${ }^{19}$-, unsere Aufsätze in möglichst angesehenen Zeitschriften unterzubringen, und konsultieren dafür Quellen wie den JCR. Aggregiert produzieren diese individuellen Optimierungsstrategien ein Ergebnis, das wir zugleich hinnehmen und beklagen: Wir lesen und verbreiten Aufsätze aus Zeitschriften mit hohem Impact Faktor, streben danach, unsere eigenen Beiträge dort zu platzieren, und sind zugleich davon überzeugt, dass unsere an weniger prominenten Stellen erschienenen Veröffentlichungen nicht wirklich schlechter sind als die an prominenter Stelle veröffentlichten Texte von Kollegen.

Der Impact Faktor wird aber nicht nur für Beförderungen, bei Berufungen, als Marketinginstrument und im Rahmen akademischer Distinktionsbemühungen verwendet, sondern auch für internationale Vergleichsstudien herangezogen. Um das zu illustrieren, verweise ich auf drei im Übrigen durchaus seriöse Abhandlungen, die auf der Grundlage der WoS-Datenbanken versuchen, internationale Vergleiche anzustellen, nationale Produktivität zu vermessen oder über den Aufstieg beziehungsweise Niedergang von Nationen etwas zu sagen. Sie alle nutzen die Daten des WoS, ohne mit auch nur einem Satz auf die Gültigkeit der dort praktizierten Klas-

15 Meyer, Rowan 1977; Baum 2011; Macdonald, Kam 2007 und 2011.

16 Vgl. Kieser 2010; Kieser 2012.

17 Willmott 2011.

18 Allerdings beträgt die Korrelation zwischen dem Impact Faktor und dem sogenannten »immediacy index " (der definiert ist als die durchschnittliche Zahl der Zitierung eines Artikel im Jahr seines Erscheinens) für 2011 nur 0,057 und ist vermutlich ein Zufallsprodukt. Im Jahr 2010 befanden sich von den »top ten «-Zeitschriften (nach Impact Faktor) nur BJS und Annual Review of Sociology in den ersten zehn Rängen des »immediacy index ", während sich im darauffolgenden Jahr 2011 ASR, BJS und AJS dort finden.

19 Power 1997; Strathern 2000; Holmwood 2010. 
sifikationen einzugehen. Philip Mirowski20 etwa zielt darauf, den Niedergang des amerikanischen Wissenschaftssystems zu belegen, und zieht dafür Daten über den rückläufigen Anteil wissenschaftlicher Veröffentlichungen aus den USA heran - ihm scheint das die Folge der zunehmenden Vermarktlichung des dortigen Wissenschaftssystems zu sein, ohne einen Gedanken darauf zu verwenden, dass das, was er beklagt, vielleicht die Folge des fehlerhaften Klassifizierens von Thomson Reuters sein könnte. Vermarktlichung mag tatsächlich eine Rolle spielen, doch höchstwahrscheinlich in einer anderen Variante als jener, die Mirowski so plausibel zu sein scheint: Um seine WoS-Datenbanken weiter wachsen zu lassen, muss Thomson Reuters Zeitschriften einbeziehen, die nicht in den USA beheimatet sind. Die Ausweitung des Erfassungshorizonts verspricht, neue Käufer des WoS zu gewinnen. Unvermeidlich geht damit aber im Verhältnis der Anteil der in den USA produzierten wissenschaftlichen Aufsätze zurück, selbst wenn sich an der »Produktivität « pro US-Wissenschaftler gar nichts ändert. Der Umstand, dass es außerhalb der USA einen riesigen und wachsenden Markt von Zeitschriften gibt, die im WoS berücksichtigt werden können, ist für ein Profitunternehmen ein starker Anreiz, der obendrein dann auch noch als Abschied vom - je nachdem - amerikanischen Provinzialismus oder Imperialismus vermarktet werden kann. Evan Schofer zieht für seinen Vergleich von " nearly 100 nations " unter anderem Daten aus dem JCR heran, die er wegen ihrer hohen Korrelation mit anderen Variablen für valide hält, obwohl nicht ausgeschlossen werden kann, dass die anderen Variablen dieselben Verzerrungen wie das WoS aufweisen. Ähnlich verfahren auch andere Vertreter des »world polity approach «. ${ }^{21}$ Der UNESCO World Social Science Report 2010 beansprucht, Analysen des »Knowledge Divides « zu liefern, scheint aber auch nicht frei von Mehrdeutigkeiten zu sein. Das dortige Porträt der »weltweiten Verteilung von sozialwissenschaftlichen Zeitschriften « beruht nicht nur auf dem WoS, sondern bezieht auch Daten aus Ulrichs und Scopus ein. Obwohl die Autoren auf Differenzen zwischen den Quellen verweisen, meinen sie, dass »das Bild in Abhängigkeit von der jeweils benutzten Datengrundlage zwar variiert, aber auf einem globalen Niveau stimmig bleibt «.22 Die Möglichkeit, dass alle drei Datenbanken dieselbe Art von Verzerrungen aufweisen, wird gar nicht in Erwägung gezogen. Es scheint, dass Mirowski, Schofer \& Co. Äpfel mit Birnen vergleichen.

Bemerkenswerterweise ist die Zahl der soziologischen Abhandlungen sehr klein, die die dem Impact Faktor zugrunde liegenden Routinen der Berechnung diskutieren. ${ }^{23}$ Jenseits der geschlossenen Zirkel der Szientometrie, die einmal von einem ihrer Gründungsväter als eine »ziemlich harte Wissenschaft « 24 bezeichnet wurde,

\section{Mirowski 2011.}

21 Schofer 2004, S. 240; Drori et al. 2003, S. 196-213.

22 Gingras, Mosbah-Natanson 2010, S. 150.

23 Aaltojärvi et al. 2008; Bjarnason, Sigfusdottir 2002; Persson 1985. Die meisten soziologischen Artikel behandeln die Naturwissenschaften und enthalten nur Nebenbemerkungen über die Sozialwissenschaften: Baldi 1998; Hargens 2000.

24 Price 1978, S. 7; vgl. Garfield 1988; Wouters, Leydesdorff 1994. 
ist das Interesse an der Erforschung dieses Felds, seiner Höhen und Tiefen, minimal und sind Studien vom Typus der social studies of science ein Desiderat. Dabei gilt es, Unterschiede zwischen Disziplinen einerseits und zwischen einzelnen Staaten und ihren Wissenschaftstraditionen andererseits im Auge zu behalten. Während in nichtwestlichen Staaten das Vertrauen in Maßzahlen ziemlich groß zu sein scheint, treten einige deutsche Professionsverbände, darunter besonders vehement die Deutsche Gesellschaft für Soziologie, gegen das Hochschulranking auf und argumentieren unter anderem mit methodischen Mängeln. ${ }^{25}$ Aber während sich die deutschsprachige Soziologie mit derartigen professionspolitischen Bekundungen zufriedenzugeben scheint, jedenfalls kaum wissenschaftliche Veröffentlichungen zum Thema zu verzeichnen sind, setzen sich Nationalökonomen, aber auch Betriebswirte eingehender mit derartigen Vermessungen und den sich dadurch wandelnden Anreizstrukturen auseinander. ${ }^{26}$ In Ländern, deren Universitäten dem Regime des New Public Management unterworfen sind, gehören regelmäßige Vermessungen der Forschungsleistungen mittlerweile zum Alltag, deren soziologische Erforschung hält damit aber nicht Schritt. In den USA untersuchen Soziologen in der Regel andere Rankings als jene, die mit dem Impact Faktor operieren. ${ }^{27} \mathrm{Ob}$ und in welcher Form Soziologen oder andere Sozialwissenschaftler an der Implementierung von Leistungsmaßzahlen für Forschung beteiligt waren oder ob die Wissenschaftsverwaltungen das aus eigenem Interesse betrieben, kann im Rahmen dieses Beitrags nicht behandelt werden, weil es dazu keine veröffentlichten Studien gibt.

Im nächsten Teil versuche ich zu zeigen, dass die Entstehung des Impact Faktors von einer großen Zahl von willkürlichen Entscheidungen begleitet war, und gehe danach genauer auf Probleme der Berechnung ein.

\section{Die Anfänge des Impact Faktors}

Der Impact Faktor ist ein Nebenprodukt der Zitationsindizes, die vom bibliografischen Unternehmer Eugene Garfield in Philadelphia, Pennsylvania, begründet wurden und ab 1963 für die Naturwissenschaften, später dann auch für die Sozial- und Geisteswissenschaften (1973 beziehungsweise 1978) veröffentlicht wurden. ${ }^{28}$ Von Anfang an analysierte Garfield mit großer Hingabe die in den Computern seiner Firma Institute for Scientific Information (ISI) archivierten Daten. Das folgenreichste Ergebnis dieser Datenexploration war der Impact Faktor, den Garfield als

25 Seit 2003 macht die Jiaotong-Universität in Shanghai mit ihrem unter anderem auf dem Impact Faktor aufbauenden Universitäts-Ranking alljährlich Schlagzeilen. Mehrere deutsche Professionsverbände sprachen sich seit 2012 dafür aus, das Hochschulranking des Centrums für Hochschulentwicklung (CHE) zu boykottieren.

26 Vgl. die Schwerpunkthefte der German Economic Review und der Perspektiven der Wirtschaftspolitik, die der Economics of the Economics Profession gewidmet ist; Schneider 2008; Fabel 2008; Kieser 2012.

27 Sauder, Espeland 2009.

28 Bensman 2007. 
Terminus bereits 1955 publik gemacht hatte. ${ }^{29}$ Eine (etwas) detaillierte(re) Auseinandersetzung mit der Entstehungsgeschichte ist lohnenswert, weil nur so gezeigt werden kann, dass das Endprodukt Impact Faktor aus Versuch und Irrtum entstand.

(1) Gemischte Motive: Anlass für die Entwicklung der Zitationsindizes war das Wachstum der Zahl wissenschaftlicher Publikationen, die selbst bibliografisch rührigsten Wissenschaftler den Überblick erschwerte. ${ }^{30}$ Anfänglich speisten die Betreiber der Zitationsindizes Literaturverzeichnisse wissenschaftlicher Aufsätze in die Datenbanken der damals noch seltenen Computer ein, um auf diesem Weg die Möglichkeit zu schaffen, die relevante Literatur für bestimmte Forschungsinteressen ausfindig zu machen. Garfields Institut ISI betrat in dem Moment den Markt des Informationshandels, als sich das Bewusstsein zu verbreiten begann, dass die Informationsexplosion - ein Ausdruck, der durch eine Konferenz der Royal Society 1948 populär gemacht wurde - nicht mehr bewältigbar sei. Die Rede vom exponentiellen Wachstum der wissenschaftlichen Veröffentlichungen lud dazu ein, nach Auswegen zu suchen. ${ }^{31}$

Die Mängel der damaligen bibliografischen Hilfsmittel ließen den Ruf nach »einem wirkungsvollen Mittel zur Verbreitung und/oder zum Finden wissenschaftlicher Literatur «32 laut werden. Schon vor dem Erscheinen des ersten Bands der Citation Indices postulierte Garfield, man könne die "Bedeutung « oder den "Einfluss « eines einzelnen wissenschaftlichen Aufsatzes anhand der Gesamtzahl der auf ihn verweisenden Zitationen messen. Bereits damals meinte Garfield, dass diese Daten auch für »soziologische Bewertungen, einschließlich Personal- und Stipendiatenauswahl « benutzt werden könnten, solange man »übermäßige und sorglose Verwendung « vermeide. ${ }^{33} 1963$ bekundeten Garfield und sein Ko-Autor Irving H. Sher, sie wären » interessiert an bestimmten $>$ Wirkungs (impact)-Faktoren, etwa wie oft ein bestimmter Aufsatz, ein bestimmter Autor oder eine bestimmte Zeitschrift zitiert wird im Vergleich mit korrespondierenden Durchschnittswerten innerhalb eines bestimmten Zitations-Index «. ${ }^{34}$ Doch die Auszählung der Zitationen allein erbrachte höchst unplausible Ergebnisse, weil einige Zeitschriften deutlich mehr Aufsätze als andere veröffentlichten. Daher schlug Garfield »einen differenzierteren Ansatz« vor: Man könne die Bedeutung, den »impact ", messen, wenn man »die Anzahl der Nennungen einer Zeitschrift " durch die »Anzahl der Artikel, die in der Zeitschrift veröffentlicht wurden «, dividiere. ${ }^{35}$ Diese Maßzahl passte besser zu den Erwartungen des Bibliografen und diente Garfield dann dazu, die Auswahl der in den Science Citation Index (SCI) aufzunehmenden Zeitschriften zu rechtfertigen. Mehrdeutigkeiten wurden allerdings nicht beseitigt, sondern Routine: Zeitschriften

29 Ebd., S. 111.

30 Vgl. Abbott 2011.

31 Für eine zeitgenössische Darstellung siehe Becker 1968.

32 Garfield 1963, S. 289.

33 Ebd., S. 290.

34 Garfield, Sher 1963, S. 199.

35 Ebd., S. 200.

Leviathan, 41. Jg., 4/2013 
fanden im SCI erst dann Berücksichtigung, wenn in ihnen eine ausreichend große Zahl häufig zitierter Aufsätze erschienen war. Wissenschaftler sind allerdings weniger an Zeitschriften als solchen interessiert als vielmehr an Aufsätzen zu ihrem jeweiligen Forschungsthema.

(2) Unterschiedliche Adressaten und Nutzer: Anfänglich waren Bibliothekare das Zielpublikum des SCI. Während Wissenschaftler relevante Literatur zu lokalisieren trachteten, wollten Bibliothekare wissen, welche Zeitschriften sie anschaffen sollten, und manche Soziologen reagierten auf die sich eröffnenden neuen Forschungsmöglichkeiten mehr oder weniger enthusiastisch: »Eine weitere Technik (die Verbreitung von Information in der Wissenschaft zu beschleunigen und wirkungsvoller zu machen), erleichtert durch die Benutzung von Hochgeschwindigkeitsrechnern, ist der Zitationsindex, der einen in die Lage versetzt, den Einfluss eines bestimmten Manuskripts über die Zeit zu verfolgen; das wird für Historiker und Wissenschaftssoziologen ebenso wertvoll sein wie für jene, die mit dem substanziellen Inhalt der zitierten Materialien befasst sind «. ${ }^{36}$ Innerhalb weniger Jahre entstand ein veritables Informationsmonopol - zuerst der SCI, dann der SSCI und der Arts \& Humanities Citation Index, die von weiteren Produkten begleitet wurden: Reference Journal Citation Index, Source Journal Citation Index, Journal Citation Reports, Current Contents, New Scientist. Weil Garfield von Beginn an Vorschläge unterbreitete wie beispielsweise jenen, wonach Zitationsindizes erlaubten, Nobelpreisträger vorherzusagen ${ }^{37}$ - und weil er nicht zögerte, eine Rhetorik der Bewertung zu kultivieren (significant, important, core, impact etc.), wurden die Daten von ISI bald mehr als nur Daten. Während der Impact Faktor selbst in den ersten drei Jahrzehnten nur wenig Aufmerksamkeit auf sich zu ziehen vermochte, ${ }^{38}$ änderte sich das ab Mitte der 1990er Jahre. Ob die, die den Impact Faktor nutzten, auch wussten, wie er berechnet wird und was diese Maßzahl auszusagen vermag und was eben nicht, darf durchaus bezweifelt werden.

(3) Experimentieren mit vorhandenen Daten: Garfields Bewunderung für die Naturwissenschaften veranlasste ihn, mit den in den Computern seines Instituts gespeicherten Daten herumzuspielen. Seine Vertrautheit mit den Debatten in den Sozialwissenschaften brachte ihn dazu, für die Interpretation auch die damals gerade entstehende Wissenschaftssoziologie heranzuziehen. 1970 sah er sich als Teil einer »neuen Gattung von Soziometristen, die sich mit historischen, soziologischen,

36 Kaplan, Storer 1968, S. 116.

37 Garfield 1973; Ashton, Oppenheim 1978.

38 Eine frühe Ausnahme ist Elkana et al. 1978. Vgl. Archambault, Larivière 2009, S. 636. Eine Suche in JSTOR nach »impact factor « in soziologischen Zeitschriften ergab nur 68 Treffer. Der erste Artikel, der den Impact Faktor diskutierte, erschien 1988. Während der folgenden beiden Jahrzehnten erschienen jährlich zwischen ein und fünf Artikel, mit einem Höhepunkt 2007, als neun Artikel veröffentlicht wurden, in denen der Impact Faktor eine Rolle spielte. Die weitgehend unterbliebene Diffusion der Erkenntnisse der Bibliometriker kann man unschwer mittels einer Zitationsanalyse der in Scientometrics erschienenen und im WoS indizierten Aufsätze überprüfen - sie fanden jenseits der geschlossenen Gesellschaft dieser Spezialisten kaum Gehör. 
ökonomischen und verhaltensbezogenen Studien von Wissenschaften und Wissenschaftlern " befassen und die er als »Wissenschaftswissenschaftler " bezeichnete. ${ }^{39}$ Seine Neigung zu statistischen Berechnungen führte zu verschiedenen Veröffentlichungen, die von führenden Wissenschaftlern jener Zeit ${ }^{40}$ und dem Begründer der Wissenschaftssoziologie freundlich aufgenommen wurden. ${ }^{41}$ In den folgenden Jahrzehnten schlugen Garfield und andere Autoren eine Vielzahl von Indikatoren vor, passten diese schrittweise an, verwarfen manche und propagierten neue - nur wenige überdauerten. Bemerkenswert ist, dass all diese Versuche strikt induktiv zustande kamen und Fachleute der jeweiligen Disziplinen den Prozess begleiteten, in welchem Ad-hoc-Entscheidungen eine große Rolle spielten.

Beim Experimentieren mit den ersten archivierten Daten, die aus der damals gerade im Aufschwung befindlichen Molekulargenetik stammten, fanden Garfield und seine Mitarbeiter einige Regelmäßigkeiten. Zuerst bemerkten sie, dass die Häufigkeiten der Zitationen eine sehr schiefe Verteilung aufwiesen: »Eine kleine Zahl von 250 Zeitschriften lieferte fast die Hälfte der 3,85 Millionen Referenzen, die das SCI im Jahre 1969 verarbeitete " ${ }^{42}$ Daraus folgerten sie, zweitens, dass es so etwas wie »core journals « gäbe, also Zeitschriften, die zum Kernbestand eines Fachs zu zählen wären: »In jeder Disziplin produzieren relativ wenige Zeitschriften etwa 90 Prozent der bedeutenden Literatur. Versuche also, 100 Prozent aller wichtigen Literatur zusammenzubekommen, würde die Zahl der auszuwertenden Zeitschriften exponentiell anwachsen lassen $« .43$

Drittens mussten Details der Berechnung des Impact Faktors fixiert werden. Bereits 1963 wurde eine Verhältniszahl vorgeschlagen, allerdings damals noch ohne irgendeine zeitliche Spezifikation: Alle bisherigen Zitatverweise wurden durch alle im SCI verzeichneten Aufsätze dividiert. Erst später, unter dem Einfluss der Arbeit zweier britischer Autoren, ${ }^{44}$ die die Daten von ISI verwendet hatten, um britische Zeitschriften zu evaluieren, behauptete Garfield, dass eine zeitliche Beschränkung des Beobachtungszeitraums die Gültigkeit nicht beeinträchtigen würde. 1972 meinte er, dass »das Verhältnis zwischen Zitationen bestimmter Jahrgänge einer Zeitschrift und der Anzahl der Artikel, die in jenen Jahren veröffentlicht wurden « ${ }^{45}$ zu ebenso gültigen Ergebnissen über die Bedeutung einer Zeitschrift führen würde wie eine Berechnung, die alle Zeiträume einbeziehe. Eine andere Entscheidung, die wieder den Einsichten der beiden britischen Autoren folgte, war noch folgenreicher. Garfield meinte, beobachtet zu haben, dass »etwa 25 Prozent aller Zitationen auf Aufsätze verweisen, die in den zwei Jahren vor dem Erscheinen des zitierenden Aufsatzes erschienen sind «. Der Faktor der aktuellen Bedeutung (»cur-

39 Garfield 1977 b, S. 158.

40 Lederberg 2000.

41 Merton 1979 a, Merton 1979 b; Merton 2000; Merton, Garfield 1986.

42 Garfield 1972, zitiert nach dem Wiederabdruck in Garfield 1977 a, S. 534.

43 Garfield 1971, S. 5.

44 Martyn, Gilchrist 1968.

45 Garfield 1977 c, S. 270. 
rent impact factor «) eliminiere bewusst »die Wirkung der meisten Superklassiker «, also jene wenigen Aufsätze einiger Berühmter, die über viele Jahre hinweg gerne zitiert werden. ${ }^{46}$ Man wird annehmen können, dass die gewählten Ad-hocBeschränkungen bei der Berechnung des Impact Faktors (nur wenige Kernzeitschriften und nur Zitate, die auf Veröffentlichungen der beiden jeweils letzten Jahre verwiesen) die Gültigkeit der Berechnung tatsächlich nicht nachhaltig verzerrten. Wir werden weiter unten sehen, dass die Übertragung dieser Einschränkungen auf andere wissenschaftliche Disziplinen, insbesondere die Sozial- und Geisteswissenschaften, weitaus weniger plausible Ergebnisse zur Folge hatte.

Davor muss noch eine weitere Entscheidung erwähnt werden, die für die Berechnung des Impact Faktors folgenreich wurde. Garfield und andere bemerkten sehr früh, dass eine bestimmte Art von Texten, sogenannte » reviews « ${ }^{47}$, also Artikel, die Literaturüberblicke boten, ohne selbst neue wissenschaftliche Erkenntnisse zu berichten, überdurchschnittlich häufig zitiert wurden. »Garfield erkannte, dass Sätze solcher Überblicksartikel implizite Indexeinträge waren und dass der Prozess der Erarbeitung eines wissenschaftlichen Index automatisiert werden konnte, indem man diese Sätze zum Ausgangsmaterial für einen solchen Index machte. Dieser Gedanke führte dann tatsächlich zur Erstellung des SCI. ${ }^{48}$ Da man Wissenschaftler bei ihrer Literatursuche unterstützen wollte, wurden diese Überblicksaufsätze nicht aus dem Korpus der einbezogenen Texte ausgeschlossen, obwohl das unter dem Gesichtspunkt, in den Zitationsindizes den Erkenntnisfortschritt zu dokumentieren, angebracht gewesen wäre. Diese Art von Texten wurde immer beliebter: Weil sie in den Zitationsindizes berücksichtigt wurden, wuchs der Anreiz, sie zu schreiben und weil die Zeitschriften, in denen diese Art von Texten erschien, in der Impact Faktor-Rangordnung nach oben kletterten, wurden sie intensiver wahrgenommen (und zitiert), und die nächste Generation von Autoren bemühte sich, solche Texte schreiben zu dürfen, weil damit der individuelle Impact Faktor vergrößert werden konnte. ${ }^{49}$ Es überrascht dann nicht, wenn es in anderen Disziplinen zu einer Nachahmung dieser Art von Besprechungszeitschriften kommt. Die Annual Reviews

46 Ebd., S. 271. Als »super-classics « wurden übermäßig häufig zitierte Aufsätze bezeichnet, die die Häufigkeitsverteilung verzerren.

47 Heute werden diese Texte von Thomson Reuters folgendermaßen definiert: »Review: An item is classified as a review if it meets any of the following criteria: it cites more than 100 references - it appears in a review publication or a review section of a journal - the word review or overview appears in its title - the abstract states that it is a review or survey « (http://admin-apps.webofknowledge.com/JCR/help/h_glossary.htm; Zugriff vom 14.06.2013).

48 Bensman 2007, S. 108

49 Eine der skurrilsten Anwendungen des Impact Faktors ist die Addition der Werte der Zeitschriften, in denen eine Person veröffentlicht hat, zu einem »individuellen « Impact Faktor. An vielen medizinischen Fakultäten/Universitäten wird man habilitiert, wenn dieser individuelle Wert einen vorab definierten Wert übertrifft. 
diverser Disziplinen zählen mittlerweile regelmäßig zu den Zeitschriften mit dem höchsten Impact Faktor. ${ }^{50}$

Heute produzieren die Computer von Thomson Reuters, ein Firmenkonglomerat, dem Garfield vor einigen Jahren sein Unternehmen ISI verkauft hatte, ${ }^{51}$ regelmäßig bibliometrische Kennzahlen, und die Mehrzahl jener, die sie verwenden, kümmert sich nicht um die Prozeduren ihrer Berechnung. Gemäß der Hilfe-Seite ist der Impact Faktor eine simple Bruchrechnung:

»Der Impact Faktor einer Zeitschrift ist die Durchschnittshäufigkeit, mit der Artikel, die in dieser Zeitschrift in den jeweils letzten zwei Jahren erschienen sind, im JCR-Bezugsjahr zitiert werden. Der Impact Faktor wird berechnet, indem die Anzahl der Zitationen im JCRBezugsjahr mit der Gesamtzahl der Artikel dividiert wird, die in den vorangegangenen zwei Jahren veröffentlicht wurde. Ein Impact Faktor 1.0 bedeutet, dass die Artikel der zwei vorangegangenen Jahrgänge im Durchschnitt einmal zitiert wurden. Ein Impact Faktor von 2.5 bedeutet, dass die Artikel der zwei vorangegangenen Jahrgänge im Durchschnitt zweieinhalb Mal zitiert wurden. Die Zitationen können aus einer einzigen Zeitschrift stammen, sind aber meistens aus verschiedenen Zeitschriften «. ${ }^{52}$

Doch wie so oft steckt der Teufel im Detail. Was ist die »Anzahl der Zitationen «? Ein gewöhnlicher Sterblicher würde antworten: alle Literaturverweise eines Aufsatzes, der in einer bestimmten Zeitschrift erschienen ist. Aus praktischen Gründen mögen Anbieter wie Thomson Reuters in seinem Web of Science nicht alle, sondern nur eine bestimmte Auswahl von Zeitschriften auswerten, aber für jede berücksichtigte Zeitschrift sollte die Zählung in gleicher Weise erfolgen. Wie noch im Detail gezeigt werden wird, ist das WoS heute weit davon entfernt, bei der Auswahl der berücksichtigten Zeitschriften die gleichen Kriterien anzuwenden, und Unterschieden der Zitierpraxis der verschiedenen Disziplinen wird nicht Rechnung getragen.

Derselbe gewöhnliche Sterbliche erlebt eine weitere Schwierigkeit, wenn er sich dem Nenner zuwendet. »Die gesamte Anzahl der Artikel der vorangegangenen zwei Jahrgänge « umfasst aber offenkundig nicht alle 247.854 Artikel und Überblicksartikel, die in den Jahren 2009 und 2010 laut Sociological Abstracts weltweit in soziologischen Zeitschriften veröffentlicht wurden, und auch nicht die 238.955 Artikel und Überblicksartikel, die laut Scopus (vom Konkurrenzunternehmen Elsevier) in demselben Zeitraum in den Sozialwissenschaften (die neben der Soziologie auch die Politikwissenschaft umfasst) erschienen sind, sondern nur jene 8.920 Arti-

50 Die erste Annual Review erschien 1932 für Biochemie, 1939 folgte Physiologie, 1947 Mikrobiologie, 1950 Psychologie, 1972 Anthropologie, 1975 Soziologie, 1998 Politische Wissenschaften und erst 2009 Ökonomie und Finanzökonomie (www.annualreviews.org/; Zugriff vom 14.06.2013).

51 Im Jahr 1992 verkaufte Garfield ISI an Thomson, einen kanadisch-britischen Konzern, der - seit 2008 zusammengeschlossen mit Reuters - ein sehr breites Portfolio aufweist: von Nachrichtendiensten für Börsen (Dow-Jones-Index) über Fachzeitschriften für den Medizin- und Pharmabereich, für Biotechnologie, Rechtswissenschaften und juristische Literatur zur Steuervermeidung bis hin zu Interessen in der Ölindustrie.

52 Siehe http://admin-apps.webofknowledge.com/JCR/help/h_impfact.htm\#impact_factor. Eine ein wenig abweichende, kürzere Erklärung des Impact Faktors findet man unter http://thomsonreuters.com/products_services/science/free/essays/impact_factor/;

Zugriffe vom 14.06.2013.

Leviathan, 41. Jg., 4/2013 
kel und Überblicksartikel, die im WoS unter "sociology « für diese beiden Jahre zu finden sind. Mit anderen Worten: Mehr als 200.000 Artikel haben jedes Jahr gar nicht die Chance, bei der Zitationszählung berücksichtigt zu werden. Doch welche Zeitschriften wurden als würdig erachtet, im WoS Berücksichtigung zu finden? Auch in diesem Fall bleibt einem nichts anderes übrig, als die Entscheidungen der Firma Thomson Reuters zu akzeptieren, die nicht gewillt ist, andere Zeitschriften als jene, die in ihrem WoS verzeichnet sind, in den Nenner ihres Impact Faktors aufzunehmen. Wie wir noch sehen werden, ist die anfängliche Begründung, nur die bedeutendsten Zeitschriften zu berücksichtigen, nicht mehr zutreffend. Vor allem in den Geistes- und Sozialwissenschaften werden neben den berühmtesten eine Menge weiterer Zeitschriften mehr oder weniger willkürlich ausgewählt.

Als Zwischenergebnis lässt sich festhalten, dass ein doppelter Elitismus die Berechnungsroutinen begleitete: Einerseits wurden die Naturwissenschaften zum Modell genommen, und andererseits wurden Textsorten, die dort weit verbreitet und anerkannt waren, wie der Überblicksartikel, jenen vorgezogen, die in den Geistes- und Sozialwissenschaften größere Bedeutung hatten, wie beispielsweise eine ausführliche Rezension eines einzelnen Buchs. Der induktiv gewonnene Impact Faktor wurde dabei schrittweise adjustiert, weil das Feld der Veröffentlichungen für die Experimentatoren rund um Garfield noch überschaubar war. ${ }^{53}$ Das Ergebnis entsprach seinen Vorerwartungen und denen seiner akademischen Berater - wohl auch, weil es ihr Bild der amerikanischen Wissenschaftslandschaft zutreffend zu zeichnen schien; später wurde die Formel ohne viel Federlesens auf andere Länder und weitere Disziplinen übertragen, ohne nochmals eine vergleichbare Adjustierung und Feinabstimmung vorzunehmen. Vertreter der erst später einbezogenen Disziplinen hatten keine vergleichbare Veto-Macht. Wie Goethes Zauberlehrling erhob Garfield späterhin mehrfach seine Stimme, um den angeblichen Missbrauch seiner Erfindung zu geißeln - doch sein Erfolg war nicht größer als jener des literarischen Gegenstücks. ${ }^{54}$

Mit einigem Recht kann man wohl behaupten, dass die monopolistische Marktmacht von ISI und der Mechanismus der self-fulfilling prophecy zusammenwirkten. Die lautstarke Verkündigung, nur die bedeutendsten Zeitschriften (anfangs der Genetik und Biologie) zu berücksichtigen, verselbstständigte sich dazu, dass jene Zeitschriften als wichtig erscheinen, die im WoS berücksichtigt sind. Obwohl, wie noch zu zeigen sein wird, die Unterschiede in den meisten sozialwissenschaftlichen Disziplinen minimal sind, ziehen die nach Impact Faktor weiter vorne gereihten Zeitschriften mehr Aufmerksamkeit auf sich als die auf hinteren Rängen platzierten.

53 Garfields umfängliche Website www.garfield.library.upenn.edu/ demonstriert, dass er in den Anfangsjahren mit vielerlei Maßzahlen experimentierte, und man darf mit einigem Recht davon ausgehen, dass jene, die er nicht weiter verfolgte, keine Unterstützung durch seine akademischen Berater fanden.

54 Beispielsweise begann Garfield einen seiner vielen Vorträge mit der Bemerkung: »I had considered as an alternative title for my talk >Citation Sanity and Insanity - the Obsession and Paranoia of Citations and Impact Factors.< Others might have preferred >Uses and Abuses of Impact Factors«; Garfield 2005. 
Verschiedene Autoren haben die Willkürlichkeit des Impact Faktor hervorgehoben, und in den folgenden Abschnitten will ich drei Aspekte näher betrachten: den Beobachtungszeitraum, die Definition der Disziplinen und die Auswahl der Zeitschriften.

\section{Die Willkürlichkeit des Impact Faktors}

\subsection{Zwei Jahre genügen nicht, zumindest nicht in der Soziologie}

Der nur zwei Jahre umfassende Beobachtungzeitraum war das Ergebnis der Untersuchung der Zitationsgewohnheiten einer kleinen Stichprobe von naturwissenschaftlichen Disziplinen, wurde danach auf alle anderen Disziplinen übertragen und wird seither im JCR präsentiert. ${ }^{55}$ Heute haben alle im Web of Science berücksichtigten Zeitschriften dieses Erbe zu schultern, das mit den Praktiken ihrer Leser und Autoren nicht in Einklang steht. Man kann das illustrieren, wenn man drei führende soziologische Zeitschriften - American Sociological Review (ASR), British Journal of Sociology (BJS) und Kölner Zeitschrift für Soziologie und Sozialpsychologie (KZfSS) - näher betrachtet. Ich habe dazu die Literaturverzeichnisse aller Hauptartikel zweier Hefte jeder der drei Zeitschriften für zwei Jahrgänge kodiert. Die Stichprobe umfasst 41 Artikel mit insgesamt 2.731 Literaturverweisen oder im Mittel 66 bibliografische Angaben je Artikel. Nur vier Prozent der Literaturverweise waren Selbstzitate (auf andere Arbeiten der Autoren des jeweiligen Artikels verweisend), wobei die Kölner hier einen leicht höheren Wert (5,5 Prozent) aufweist. Diese geringe Rate der Selbstzitation macht es unnötig, diese ansonsten gerne diskutierte Verzerrung eingehender zu behandeln. Rund die Hälfte der Literaturverweise betraf Zeitschriftenaufsätze, und die andere Hälfte ging an alle anderen Textsorten (Bücher, Buchkapitel, Tageszeitungen, Internetquellen etc.). Die 1.388 zitierten Zeitschriftenartikel erschienen in irgendeiner Zeitschrift, während der Impact Faktor bekanntermaßen nur aus den im SSCI erfassten Zeitschriften berechnet wird. Ich unterließ eine Überprüfung, welcher Anteil der Zitationen auf Zeitschriften entfiel, die im SSCI enthalten sind, ${ }^{56}$ auch weil ein anderer Befund weitaus dramatischer war: Nur 5,6 Prozent aller Zitationen entfielen auf Artikel, die in den zwei davorliegenden Jahren erschienen waren. Dieser Wert bleibt weit unter dem von Garfield etablierten Grenzwert von 25 Prozent. ${ }^{57}$ In Disziplinen wie der Soziologie folgt die Zitationspraxis offenkundig anderen Regeln als in (manchen der) "harten "Wissenschaften. Das bedeutet für die drei hier analysierten - und mit größter Wahrscheinlichkeit auch für alle anderen - soziologischen Zeitschriften, dass der Impact Faktor nur auf der Basis von weniger als einem Zwanzigstel der Zitationen berech-

55 Die weite Verbreitung des JCR ist wohl auch das Ergebnis der Preispolitik: Der Abonnementpreis für JCR ist deutlich geringer als jener für das Gesamtpaket WoS (Auskunft eines Mitarbeiters von Thomson Reuters anlässlich einer Schulung im November 2012).

56 Chi berichtet für politikwissenschaftliche Zeitschriften, dass 53 Prozent der Literaturverweise auf Zeitschriften, die im SSCI gelistet sind, verwiesen; Chi 2012, S. 442.

57 Garfield 1977 c, S. 271.

Leviathan, 41. Jg., 4/2013 
Tabelle 1: Zitationspraktiken ausgewählter soziologischer Zeitschriften

\begin{tabular}{|c|c|c|c|c|c|c|c|c|c|c|}
\hline & & & & \multicolumn{4}{|c|}{ Zitationsfenster: 2 Jahre } & \multicolumn{2}{|c|}{ Selbst-Zitationen } & \multirow{2}{*}{$\underset{\text { nen }}{\sum \text { Zitatio- }}$} \\
\hline Zeitschrift & Jg. $(\mathrm{H})$ & Artikel & Lit/Art & Artikel & $\%$ & andere & $\%$ & & $\%$ & \\
\hline ASR & $77(4+5)$ & 14 & 91,2 & 72 & 5,6 & 55 & 4,3 & 42 & 3,3 & 1.277 \\
\hline BJS & $62(3+4)$ & 17 & 54,2 & 63 & 6,8 & 62 & 6,7 & 35 & 3,8 & 922 \\
\hline KZfSS & $64(1+2)$ & 10 & 53,2 & 23 & 4,3 & 31 & 5,8 & 29 & 5,5 & 532 \\
\hline$\sum$ bzw. Mittel & & 41 & 66,2 & 158 & 5,6 & 148 & 5,6 & 106 & 4,2 & 2.731 \\
\hline & & & & \multicolumn{4}{|c|}{ Zitationsfenster: 3 bis 5 Jahre } & & & \\
\hline Zeitschrift & & & & Artikel & $\%$ & andere & $\%$ & & & \\
\hline ASR & & & & 154 & 12,1 & 85 & 6,7 & & & \\
\hline BJS & & & & 69 & 7,5 & 73 & 7,9 & & & \\
\hline KZfSS & & & & 51 & 9,6 & 39 & 7,3 & & & \\
\hline$\sum$ bzw. Mittel & & & & 274 & 9,7 & 197 & 7,3 & & & \\
\hline & & & & \multicolumn{4}{|c|}{ Zitationsfenster: älter als 5 Jahre } & & & \\
\hline Zeitschrift & & & & Artikel & $\%$ & andere & $\%$ & & & \\
\hline ASR & & & & 554 & 43,4 & 357 & 28,0 & & & \\
\hline BJS & & & & 197 & 21,4 & 458 & 49,7 & & & \\
\hline KZfSS & & & & 205 & 38,5 & 183 & 34,4 & & & \\
\hline$\sum$ bzw. Mittel & & & & 956 & 34,4 & 998 & 37,3 & & & \\
\hline
\end{tabular}

Erläuterungen: Lit/Art: Durchschnitt der Zahl der Literaturverweise je Artikel;

Zitationsfenster: Zahl der Zitierungen des Artikel des jeweiligen Jahrgangs, die auf Werke der davorliegenden 2 beziehungsweise 3-5 Jahre oder auf frühere Jahre verweisen;

Selbstzitationen: Zitationen, die auf eigene Veröffentlichungen verweisen;

$\sum$ bzw. Mittel: Spaltensumme beziehungsweise Mittelwerte;

ASR: American Sociological Review;

BJS: British Journal of Sociology;

KZfSS: Kölner Zeitschrift für Soziologie und Sozialpsychologie.

Quelle: Eigene Auszählung.

net wird (weil ja für dessen Berechnung nur die kleine Zahl der im WoS aufgenommenen Zeitschriften herangezogen wird). Einige der 41 ausgewerteten Artikel zitierten keinen einzigen Text aus dem zweijährigen Beobachtungszeitraum. Der größte Teil der zitierten Literatur verweist auf Texte, die fünf oder mehr Jahre vor der zitierenden Quelle erschienen sind (siehe Tabelle 1). 
Zugunsten der Praxis von SSCI und JCR könnte jemand ins Treffen führen, dass die kleine Stichprobe von elf Prozent ausreichend sei, um zu gültigen Ergebnisse zu kommen. In Verfolgung dieser Idee müsste man argumentieren, dass in allen sozialwissenschaftlichen Aufsätzen auch auf die aktuelle Forschungsliteratur Bezug genommen wird und die Auswertung nur dieser Zitationen eine hinreichend breite Basis für die Vermessung von Qualitätsunterschieden zwischen den Zeitschriften darstelle. Mit Blick auf die Zitiergewohnheiten von Sozialwissenschaftlern wird man das aber bezweifeln können.

Die Daten in Tabelle 1 zeigen, dass Soziologen jüngst erschienene Veröffentlichungen nicht systematisch älteren vorziehen; gelegentlich entwickeln sich unsere Debatten eben deutlich langsamer als beispielsweise in der Zellbiologie, und grundlegende Aufsätze sind meist länger haltbar. Zitationen anderer Textsorten, beispielsweise Bücher, und älterer Zeitschriftenaufsätze sind in der Soziologie die Regel und nicht die Ausnahme.

Verschiedene Kritiker und einige Verteidiger des SSCI haben darauf verwiesen, dass der fünf Jahre umfassende Impact Faktor, über den JCR routinemäßig auch berichtet, die aussagekräftigere Maßzahl wäre. Der (kumulierte) Anteil der Zitationen, die in diesen Zeitraum fallen, ist im Fall der drei untersuchten Zeitschriften mit 15 Prozent aber nur unmerklich größer als der Wert für die Zwei-Jahres-Periode ( $\mathrm{da}$ in die Zählung hier wiederum Zeitschriften eingingen, die nicht im WoS indiziert sind, liegt der WoS-Wert deutlich darunter).

Diese recht simple Überprüfung der Validität steht im Einklang mit dem, was Soziologen wissen (könnten), was aber in der öffentlichen Debatte über die Verwendung von Kennzahlen regelmäßig verloren geht. Bemerkenswerterweise schieben Herausgeber und Verlage nahezu aller soziologischen Zeitschriften, die im WoS/ SSCI berücksichtigt werden, dieses Wissen immer dann beiseite, wenn der Impact Faktor ihren Marketingbemühungen zupass kommt.

\subsection{Was ist eine Disziplin?}

Eine zweite Besonderheit, die zu Willkür führt, wurzelt in der Definition von Disziplinen. Der SSCI umfasst 41 verschiedene Disziplinen (»subjects «; SCI rund 170), doch es gelingt nicht, eine nachvollziehbare Rechtfertigung ihrer Zusammenstellung ausfindig zu machen. $\mathrm{Zu}$ den Sozialwissenschaften zählen die traditionellen Disziplinen wie Anthropologie, Geografie, Geschichtswissenschaft, Rechtswissenschaft, politische Wissenschaft, Soziologie etc. (wobei die Berücksichtigung von »history « und »law « dem US-amerikanischen Verständnis von »social sciences « verpflichtet ist). Die Psychologie zerfällt in zehn Teilgebiete plus Psychiatrie, während die Ökonomie nur als ein »subject " aufscheint. Des Weiteren finden wir Spezialdisziplinen und interdisziplinäre Felder wie »ethnic studies ", »family studies « etc., aber auch weniger bekannte Gebiete wie »ergonomics ", »planning \& development ", » rehabilitation ", "substance abuse « etc. Insgesamt sind rund 2.700 Zeitschriften im SSCI (mit rund 1.000, die zwei- oder mehrfach zugeordnet sind): 350 in der Ökonomie, 220 in "education " und 608 in den zehn Teilgebieten der Psychologie. Andere 
»subjects « sind so klein wie » history of social sciences « (mit 27 Zeitschriften $)^{58}$ oder "social sciences, mathematical methods " (43), aber auch breitere Gebiete wie beispielsweise "social issue", sind nur mit vergleichsweise wenigen Titeln vertreten (35), während unter "social sciences, interdisciplinary « und "rehabilitation " eine weit größere Zahl (84 und 64) als in deutlich besser definierten oder etablierten Feldern zu finden sind: »demography « (24), "gerontology « (30), »urban studies « (36) sowie die weniger deutlich ausdifferenzierten Gebiete »transportation " (23) und »substance abuse« (29).

Die disziplinäre Klassifikation einzelner Zeitschriften ist fragwürdig. Beispielsweise ist Poetics in Soziologie zu finden, Economy and Society ist sowohl in Ökonomie als auch Soziologie vertreten, hingegen findet man Minerva gleich in drei Gebieten (»education \& educational research ", "history \& philosophy of science « und »social sciences, interdisciplinary ", aber nicht in » history of social sciences «), und New Left Review in zwei ("political science " und "social sciences, interdisciplinary «). Actes de la recherche en sciences sociales ist nur in "social science, interdisciplinary " gelistet, während Human Relations dort und unter "management " $\mathrm{zu}$ finden ist. Eine den Zuordnungen zugrunde liegende Logik ist nicht zu erkennen. Die Aufnahme von Zeitschriften in die Restkategorie »social science, interdisciplinary « lässt sich wohl nur im Zusammenhang mit den Marketing-Bemühungen der Firma Thomson Reuters deuten: Im Jahr 2000 wurden dort 55 Titel angeführt, im Jahr 2012 waren es bereits 92. Der Effekt arbiträrer Erweiterung des WoS-Zeitschriftenpools - durch Aufnahme von Zeitschriften wie South African Journal for Research in Sport, Physical Education and Recreation, New Perspectives on Turkey, das esthnische Journal of the Humanities and Social Sciences, die kolumbianische Revista de Estudios Sociales, die mexikanische Perfiles Latinoamericanos und andere mehr - ist die Vergrößerung des Zählers (und damit des Impact Faktors) der Zeitschriften aus jenen »subjects ", die für Disziplinen stehen, weil in den randständigen Zeitschriften die prominenteren zitiert werden, das Umgekehrte aber nicht zutrifft. ${ }^{59}$

Die Auswahl steht weder in einem erkennbaren Zusammenhang mit der Zahl der Zeitschriften in verschiedenen Disziplinen, noch folgt sie dem Kriterium der Reputation, obwohl in der Selbstdarstellung des WoS gerade letzteres behauptet wird. Man kann das an einem Vergleich der Bandbreiten der Impact Faktoren verschiedener Disziplinen deutlich machen. Beispielsweise ist die psychologische Zeitschrift mit dem höchsten Impact Faktor Behavioral and Brain Sciences $(21,9)$, gefolgt von Annual Review of Psychology (18,3), doch die Zeitschriften auf den Rängen ab 200

58 Man gewinnt den Eindruck, dass die Platzierung einzelner Zeitschriften durch eine maschinelle Zuordnung erfolgte: Wenn »history* "im Titel, dann ordne die Zeitschrift dem subject » history of social sciences « zu. Zeitschriften wie History of the Family und Business History gehören mit Sicherheit nicht in dieses Feld und haben auch sonst nichts gemeinsam.

59 Letztere Behauptung kann im Journal Citation Reports für jede Zeitschrift anhand der »Cited Journal data table« überprüft werden. Das obige Resümee wurde anhand der genannten fünf Zeitschriften überprüft. 
(insgesamt 406) haben alle einen Impact Faktor, der kleiner als 1,8 ist. In der Ökonomie ist das Journal of Economic Literature $(7,4)$ auf Rang 1, und ab Rang 40 unterschreiten insgesamt 260 Zeitschriften den Wert von 1,8. Der Impact Faktor der anthropologischen Zeitschriften erstreckt sich von 4,5 bis 0,076, einen Impact Faktor höher als 1,8 erzielen nur die ersten elf Zeitschriften. Die Mediane der 41 Teilbereiche der Sozialwissenschaften reichen von 1,8 für " psychology, biological « bis zu mageren 0,3 für »cultural studies « und »history « 60

In den frühen Tagen der Zitationsindizes wurde argumentiert, dass nur jene Zeitschriften Aufnahme finden sollten, die eine hinreichend hohe Reputation besitzen, die in der Zahl der auf sie entfallenden Zitationen ausgedrückt wird, und alle jene, die einen (pragmatisch definierten) Grenzwert nicht überschreiten, ausgeklammert bleiben sollten. ${ }^{61}$ Seit Thomson Reuters Eigentümer von ISI ist, folgt die Auswahl der Zeitschriften nicht mehr allein der Reputation, sondern einer gemischten Strategie. Worüber die WoS-Internetseite auch unumwunden Auskunft gibt:

»Alle sozialwissenschaftlichen Zeitschriften werden derselben sorgfältigen Bewertung unterzogen wie in den Naturwissenschaften. Die Veröffentlichungsstandards, die Richtlinien der Herausgeber, die internationale Vielfalt und die Zitationsdaten werden alle berücksichtigt. Standardzitationsmaßstäbe, sowohl auf Autoren- als auch auf der Ebene der Zeitschrift, werden analysiert, wobei man im Auge hat, dass die allgemeine Zitationsrate in den Sozialwissenschaften niedriger ist als in den Naturwissenschaften. Regionalstudien haben eine besondere Bedeutung in den Sozialwissenschaften, da Themen lokalen Interesses öfter im Fokus der Studien der Sozialwissenschaftler stehen als globale Interessen «. ${ }^{62}$

Die willkürliche Aufnahme bestimmter Zeitschriften und deren Zuordnung zu beliebig definierten Pseudo-Disziplinen (»subject category«) beeinflussen jedoch den Impact Faktor umso stärker, je kleiner die Zahlen der »citations « sind.

\subsection{Europäische Zeitschriften im SSCI}

Die Daten des WoS, die im JCR publik gemacht werden, beeindrucken nicht nur unterschiedliche Nutzergruppen, sondern werden auch in internationalen Ver-

60 Es ist angebracht, die Nicht-Spezialisten unter den Lesern darauf hinzuweisen, dass in den Naturwissenschaften die Mediane und die Impact Faktoren einzelner Jahre größer sind. In der Biochemie beträgt der Median 2,34, in der Mathematik hingegen nur 0,58; die Zeitschrift mit dem höchsten Impact Faktor ist Cell mit 32,406, während im SSCI Behavioral and Brain Sciences mit 21,952 den höchsten Impact Faktor aufweist, das Journal of Economic Literature (Rang 11) hat einen Impact Faktor von 7,432, und die höchstrangige soziologische Zeitschrift American Sociological Review befindet sich mit einem Impact Faktor von 3,693 auf Rang 91.

61 Die ursprüngliche Begründung für die Beschränkung auf bestimmte Zeitschriften berief sich auf bestimmte »Gesetze« über exponentiell abnehmende Erträge der Erweiterung einer Suche nach Referenzen in wissenschaftlichen Zeitschriften (Bradford's law of scattering), über die Anzahl von Publikationen eines Autors und der Anzahl von Autoren mit gleich hoher Zahl an Publikationen (Lotka's law) sowie »Garfield's law of concentration «. Die Botschaft all dieser Verteilungsmuster war, dass man sich in der Bibliometrie auf einen Kern von Zeitschriften beschränken kann, weil man damit einen hinreichend großen Anteil der gesamten Literatur bibliografisch erfasse.

62 Testa 2012.

Leviathan, 41. Jg., 4/2013 
gleichsstudien verwendet. Um deren mangelnde Eignung für die Analyse nationalstaatlicher Wissenschaftsproduktivität aufzuzeigen, soll im Folgenden die Repräsentanz der »europäischen Soziologie « im SSCI etwas genauer untersucht werden. Da es nicht leichtfällt, das Phänomen »europäische Soziologie zutreffend einzugrenzen, sind Autoren genötigt, sich mithilfe verschiedener Datensammlungen zu behelfen. Üblicherweise werden die folgenden benutzt: die Sociological Abstracts (SA) von ProQuest, Scopus von Elsevier, das Web of Science von Thomson Reuters und gelegentlich auch Ulrichs Verzeichnis wissenschaftlicher Zeitschriften. Während SA darauf zielt, möglichst alle Zeitschriften (und mittlerweile auch weitere Publikationsformen) einzubeziehen ${ }^{63}$ (aber selbst keine Auswertung der gesammelten Daten vornimmt), zeichnen sich Scopus und WoS dadurch aus, dass sie neben den bibliografischen Angaben auch statistische Daten generieren.

Beschränkt man eine Suche in den SA auf jene Länder, die man im weitesten zu Europa zählen kann, findet man für ein einzelnes Jahr in Summe rund 10.000 Artikel bibliografiert. Der SSCI ist deutlich selektiver und verzeichnet für dasselbe Jahr nur 4.370 » articles « und 108 Überblicksartikel (»reviews «) unter dem »subject « Soziologie. Elseviers Scopus verfolgt eine deutlich andere Strategie und sammelt in dem weitaus breiter definierten Bereich der »social sciences « insgesamt mehr als 125.000 »articles « und »reviews " (alle Daten für 2010). ${ }^{64}$

Alle drei Datenbanken ermöglichen eine Suche nach regionaler Herkunft. Das Europa sieht jeweils sehr unterschiedlich aus. Der SSCI und sein Pendant JCR erlauben es, Zeitschriften nach dem "country of publication « zu selektieren. ${ }^{65} \mathrm{Im} \mathrm{Jahr}$ 2010 sind (inklusive Mehrfachzuordnungen) 142 Zeitschriften dem »subject « Soziologie zugeordnet. In 17 europäischen Staaten erscheinen angeblich 59 Zeitschriften (siehe Tabelle 2). Einige der Zeitschriften sind sowohl anderen Disziplinen (die von »hospitality, leisure, sport \& tourism « bis zu vertrauteren Gebieten wie Anthropologie, Linguistik, Ökonomie etc. reichen) als auch der Soziologie zugeordnet. Lässt man alle Doppel- und Mehrfachzuordnungen unberücksichtigt, reduziert sich die Zahl der nur unter »sociology « klassifizierten Zeitschriften auf 36.

63 Laut der Website von Sociological Abstracts werden »over 1800 serials, over $40 \%$ of titles are published outside North America « berücksichtigt, wobei alle Teilgebiete der Soziologie und ausgewählter »content from such other disciplines as anthropology, social psychology, demography, education, criminology, penology, and political science « einbezogen werden.

64 Ein offensichtlicher Mangel all dieser Statistiken ist die fehlende Berücksichtigung von Büchern. Engels et al. 2012 liefern Hinweise auf die Verteilung der Textsorten für die Soziologen der flämischen Universitäten in Belgien. Im Untersuchungszeitraum fanden sie 3.000 Artikel, 123 Beiträge in Sammelbänden, zehn herausgegebene Bücher und sieben Monografien, verfasst von Soziologen. Vgl. Ward 2010.

65 Andere bibliometrische Berichte von Thomson Reuters, wie Essential Science Indicators oder Journal Performance Indicators, standen mir in den von mir benutzen Bibliotheken für die vorliegende Analyse nicht zur Verfügung. 
Tabelle 2: Soziologische Zeitschriften in drei verschiedenen Datenbanken, nach Ländern

\begin{tabular}{|c|c|c|c|c|}
\hline Land & JCR manuell & WoS/JCR & Scopus & Ulrichs \\
\hline International & 37 & & & \\
\hline USA & 59 & 62 & 134 & 200 \\
\hline Großbritannien & 17 & 36 & 198 & 144 \\
\hline Deutschland & 5 & 5 & 25 & 22 \\
\hline Kanada & 4 & 4 & 10 & 21 \\
\hline Polen & 3 & 2 & 1 & 2 \\
\hline Australien & 2 & 1 & 7 & 22 \\
\hline Kroatien & 2 & 2 & 5 & 3 \\
\hline Frankreich & 2 & 3 & 15 & 12 \\
\hline Spanien & 2 & 2 & 12 & 4 \\
\hline Brasilien & 1 & 1 & 10 & 4 \\
\hline China/Hong Kong & 1 & 1 & 1 & 0 \\
\hline Tschechische Republik & 1 & 1 & 1 & 3 \\
\hline Indien & 1 & 1 & 4 & 8 \\
\hline Irland & 1 & 1 & 3 & 0 \\
\hline Japan & 1 & 1 & 1 & 0 \\
\hline Litauen & 1 & 1 & 1 & 2 \\
\hline Mexiko & 1 & 1 & 1 & 6 \\
\hline Niederlande & 1 & 9 & 47 & 13 \\
\hline Neuseeland & 1 & 0 & 0 & 6 \\
\hline Rumänien & 1 & 1 & 2 & 3 \\
\hline Russische Föderation & 1 & 1 & 5 & 4 \\
\hline Slowakei & 1 & 1 & 1 & 2 \\
\hline Schweden & 1 & 1 & 3 & 7 \\
\hline Schweiz & 1 & 2 & 3 & 1 \\
\hline Österreich & 0 & 0 & 2 & 2 \\
\hline Belgien & 0 & 1 & 2 & 6 \\
\hline Chile & 0 & 0 & 1 & 2 \\
\hline Kolumbien & 0 & 0 & 3 & 3 \\
\hline Dänemark & 0 & 0 & 1 & 4 \\
\hline Ungarn & 0 & 0 & 3 & 1 \\
\hline Israel & 0 & 0 & 0 & 1 \\
\hline Italien & 0 & 0 & 10 & 1 \\
\hline Malaysia & 0 & 0 & 2 & 2 \\
\hline Norwegen & 0 & 1 & 3 & 1 \\
\hline Philippinen & 0 & 0 & 2 & 0 \\
\hline Slowenien & 0 & 0 & 2 & 1 \\
\hline Südafrika & 0 & 0 & 2 & 2 \\
\hline Süd-Korea & 0 & 0 & 3 & 2 \\
\hline Taiwan & 0 & 0 & 5 & 0 \\
\hline Türkei & 0 & 0 & 1 & 3 \\
\hline Venezuela & 0 & 0 & 2 & 3 \\
\hline Summe & 148 & 142 & 531 & 517 \\
\hline
\end{tabular}

Quellen: WoS/JCR (2009-11); Scopus, Ulrichs und JCR manuell (2011); eigene Auszäblung.

Eine Überprüfung dieser angeblich europäischen Zeitschriften brachte Befremdliches zutage: Praktisch in allen Fällen ist das "country of publication " nämlich das Land, in dem der Verlag beheimatet ist, der die betreffende Zeitschrift verlegt. Bei- 
spielsweise gehört das Journal of Sociology angeblich nach Großbritannien, aber tatsächlich ist dort nur das Verlagshaus Sage beheimatet, während die Zeitschrift in Australien gemacht wird, ist sie doch das offizielle Organ von The Australian Sociological Association (TASA).

Eine Inspektion der Webseiten aller untersuchten Zeitschriften zeigte die Unmöglichkeit, manche sinnvoll einem bestimmten Nationalstaat zuordnen zu können, weil Herausgeber in verschiedenen Staaten leben; ich klassifizierte alle diese Zeitschriften als »international «, in der Summe waren es $37 .{ }^{66}$ Die irreführenden Angaben über die »Nationalität « der Zeitschriften reduzierte die Zahl der britischen und der Zeitschriften, die angeblich in den Niederlanden beheimatet sind, während die Zahl der US-amerikanischen Zeitschriften nur unwesentlich kleiner wurde.

Wie erwähnt, verfolgt Thomson Reuters eine gemischte Strategie bei der Auswahl der in das WoS einbezogenen Zeitschriften. Im Fall der Soziologie heißt das, dass die ersten 20 von den rund 140 Zeitschriften wohl wirklich prominent sind. Wählt man als Grenzwert einen Impact Faktor > 1,8, dann übertrafen ihn 2010 nur 14 Zeitschriften. Auf den hinteren Rängen herrscht Beliebigkeit. Ob die polnische Eastern European Countryside (2010 Impact Faktor 0,053), Filosofija - Sociologija (2010 Impact Faktor 0,179) aus Litauen und das in Wien redigierte Innovation The European Journal of Social Science Research (2010 Impact Faktor 0,326) zum Kern der wichtigsten europäischen Zeitschriften gehören, scheint fraglich, zumindest solange andere von vergleichbarer oder gar höherer Reputation nicht aufgenommen werden.

Nach 1989 fanden zunehmend Zeitschriften aus dem früheren Sowjeteuropa Berücksichtigung, was zu einer schiefen Verteilung der europäischen Zeitschriften im SSCI führte: Die 59 von mir überprüften Zeitschriften aus Europa (ohne die » internationalen «) verteilen sich folgendermaßen auf Länder: 17 haben Herausgeber, die in Großbritannien zuhause sind, fünf in Deutschland, drei in Polen, je zwei in Kroatien, Frankreich und Spanien und jeweils eine Zeitschrift aus Belgien, Dänemark, Irland, Litauen, den Niederlanden, Norwegen, Rumänien, der Russischen Föderation, Slowakei, Schweden, Schweiz und der Tschechischen Republik. (Einige europäische Länder sind überhaupt nicht vertreten: Bulgarien, Finnland, Griechenland, Italien, Portugal, Österreich.) Oder: neun aus dem früheren Westen Europas und acht aus dem früheren Osten. Diese Verteilung korrespondiert in etwa mit der Zahl der Staaten und eventuell auch noch den Bevölkerungszahlen, vermutlich aber nicht der Zahl der Soziologen dies- und jenseits des ehemaligen Eisernen Vorhangs. Allerdings fehlen Daten über die Zahl der Soziologen in Europa. Eine näherungsweise Schätzung erlauben die Angaben jener nationalen Soziologenverbände, die entweder Mitglieder der European Sociological Association (ESA) oder der International Sociological Association (ISA) sind $(\mathrm{n}=33)$. Eine Extrapolation der Zahl der Soziologen je 100.000 Einwohner jener Länder, über die Daten gefunden werden konnten $(\mathrm{n}=16)$, auf Europa insgesamt ergibt eine Zahl von mindestens 14.000 Soziologen in Europa. Diese Schätzung würde Europa auf die gleiche Stufe mit den

66 Beispiele: Theory and Society, Poetics, Discourse \& Society, Qualitative Research, Acta Sociologica, European Sociological Review etc. 
USA stellen. ${ }^{67}$ Folgen wir dieser Annahme und unterstellen wir einen vergleichbaren Grad der Institutionalisierung, könnten wir im Anschluss an einen Hinweis von Abbott ${ }^{68}$ erwarten, dass wir dies- und jenseits des Atlantiks etwa dieselbe Zahl an soziologischen Zeitschriften vorfinden. Eine Suche im Ulrichs bestätigt das: Von den 538 soziologischen Zeitschriften, die dort verzeichnet sind, entfallen ungefähr gleich viele auf Europa (244) und Nordamerika (USA und Kanada: 221). ${ }^{69}$ Doch auch hier muss bedacht werden, dass die Klassifikation der "Nationalität " wissenschaftlicher Zeitschriften höchst fragwürdig ist. Scopus, WoS/JCR und Ulrichs klassifizieren die Publikationsländer durch die Verlagsorte. In Großbritannien und in den Niederlanden scheinen daher viele Zeitschriften beheimatet zu sein (was aber nur darauf zurückzuführen ist, dass Verlage wie Elsevier, Wiley, Sage dort ihre Firmenzentralen haben), während andere Staaten in diesen Datensammlungen durch keine (Bulgarien, Griechenland, Portugal) oder deutlich weniger Zeitschriften (wie im Fall Italiens) vertreten sind.

Ein Vergleich, der sich auf die Zahl der Veröffentlichungen bezieht, ergibt ein weniger passendes Bild. Scopus offeriert die Möglichkeit, die regionale Herkunft der »citable documents « festzustellen. Wir können nur hoffen, dass die Klassifikation der Adressen der Autoren (der »citable documents «) weniger fehleranfällig ist als die der Zeitschriften. Demnach wären im ersten Jahrzehnt des 21. Jahrhunderts 93 Prozent der »documents « dem Westen Europas zuzuzählen, und nur sieben Prozent hätten ihren Ursprung im Osten Europas.

Da es keinerlei Hinweise darauf gibt, dass die Soziologen aus dem früheren Sowjeteuropa weitaus mehr bemerkenswerte Aufsätze produzieren als die Westeuropäer, darf man wohl behaupten, dass die Entscheidung von Thomson Reuters, nahezu die gleiche Zahl von Zeitschriften aus dem Westen wie dem Osten Europas zu berücksichtigen, wenig mit wissenschaftlicher Qualität zu tun hat, sondern Marktinteressen geschuldet ist. Um den Markt der Transformationsländer zu erobern, kam Thomson Reuters diesen Staaten entgegen und adelte ihre oftmals außerhalb des Herkunftslands nahezu unbekannten Zeitschriften durch Aufnahme in den vorgeblich nach Reputation selektierenden SSCI.

Die Rhetorik des WoS, in der ständig von "core ", "significance ", " recognition ", »impact « etc. die Rede ist, steht in einem markanten Gegensatz zu der offensichtlichen Beliebigkeit der Datengrundlage.

67 Kirchner gibt die Zahl der ASA-Mitglieder mit 13.000 an (Kirchner 2004), Europa Publication 2012 und die ASA-Webseite nennen 14.000.

68 Abbott schätzt, dass auf jeweils 150 Wissenschaftler die Gründung einer neuen Zeitschrift kommt (Abbott 2011).

69 Der Rest verteilt sich auf Australien und Neuseeland (28), Asien (20), Südamerika (20) und Afrika (5) (http://ulrichsweb.serialssolutions.com; Zugriff vom 01.05.2012). 


\section{Die sachliche Belanglosigkeit des Impact Faktors}

Der JCR offeriert mehr als eine Maßzahl für die im WoS erfassten Zeitschriften. Der Impact Faktor und der Fünf-Jahres-Impact Faktor sind die am häufigsten benutzten Kennzahlen. Die soziologische Zeitschrift mit dem höchsten Fünf-JahresImpact Faktor ist die American Sociological Review (2010: 5,8; 2011: 5,7; zum Vergleich der Zwei-Jahres-Impact Faktor: 4,4 in 2011), gefolgt vom American Journal of Sociology (2010: 5,1; 2011: 3,1; Zwei-Jahres-Impact Faktor 2011: 3,2) und der Annual Review of Sociology (2010: 5,03; 2011: 5,8; Zwei-Jahres-Impact Faktor 2011: 4,4). Auf Rang 8 folgt die erste europäische Zeitschrift, das British Journal of Sociology, mit einem Fünf-Jahres-Impact Faktor von 2,8 im Jahr 2010 (2011: 2,1; Zwei-Jahres-Impact Faktor 2011: 1,7).

Eine Berechnung des Mittelwerts des Zwei-Jahres-Impact Faktors über einen längeren Beobachtungszeitraum (2000 bis 2011) hinweg sollte ein glatteres Ergebnis liefern: Wiederum ist die Zeitschrift mit dem höchsten Mittelwert die American Sociological Review mit 3,2, gefolgt von der Annual Review of Sociology mit einem Wert von 2,9 und dem American Journal of Sociology mit 2,8. Die nächsten zehn Zeitschriften zeigen nur noch sehr geringe Differenzen (von 1,78 bis 1,39). Noch weiter hinten verschwinden die Unterschiede nahezu völlig. Die Zeitschriften, die die Ränge 14 bis 32 einnehmen, liegen im Bereich von 1,387 bis 1,024. Ab dem Rang 33 liegt der Mittelwert über zwölf Jahre bereits unter $1 .^{70}$

Mit anderen Worten: Ein durchschnittlicher Artikel, der in einer der drei besten Zeitschriften veröffentlicht wurde, wird innerhalb der folgenden beiden Jahre im Durchschnitt drei Mal zitiert, Beiträge der nächsten 28 Zeitschriften erzielen eine Resonanz von einer Erwähnung pro Jahr, und der Rest der mehr als 100 im SSCI erfassten Zeitschriften erzielt eine Aufmerksamkeit von weniger als einer Zitation. Dieses Bild steht in einem markanten Gegensatz zu den Alltagserfahrungen von Soziologen, die über eine (wenn auch nur moderat) bessere Wahrnehmung ihrer Veröffentlichungen berichten (können). Die Diskrepanz ist das Resultat schiefer Verteilung der Zitationen (einige Artikel werden häufig, die meisten selten oder gar nicht zitiert), aber auch Folge der willkürlichen Kriterien, die der Berechnung des Impact Faktors zugrunde liegen. Man kann das anhand der Berechnung eines ein wenig anders komponierten Impact Faktors demonstrieren.

»Offiziell « ist der Impact Faktor einer Zeitschrift A im Bezugsjahr X die Verhältniszahl zwischen der Summe der Zitatverweise der im WoS im Jahr X berücksichtigten Zeitschriften auf Artikel, die in den beiden davorliegenden Jahren (X-1 + X-2) in der Zeitschrift A erschienen sind, dividiert durch die Summe der Artikel, die in der Zeitschrift A in diesen beiden Jahren (X-1 + X-2) erschienen sind (siehe Tabelle 3). Angesichts der Langsamkeit, mit der Soziologinnen und Soziologen die Veröf-

70 Ignoriert man die willkürliche Berechnung des Impact Faktors mit vier Kommastellen (die nur eingeführt wurde, um ex aequo-Platzierungen zu minimieren) und begnügt sich mit zwei Kommastellen, ist das Ergebnis für die ersten 42 Titel erhellend: Während die ersten drei Ränge von den angeführten Zeitschriften eingenommen werden, entfallen auf die folgenden Ränge jeweils 3, 3, 6, 7, 4, 2 gleichrangige Zeitschriften, und Rang 13 teilen sich gar 13 Zeitschriften. 
fentlichungen ihrer Kollegen wahrnehmen, wäre es angebracht, den Beobachtungszeitraum zumindest um ein Jahr nach hinten zu verlegen, wenn man den Betreibern des Impact Faktor-Gewerbes ein Interesse an der Validität ihrer Daten unterstellen würde. Um diesen Impact Faktor zu berechnen, würde man also nicht die Zitate aus dem das Bezugsjahr 1 auf die Jahre - 1 und -2 heranziehen, sondern die Jahre -2 und -3 der Berechnung zugrunde legen. Anhand von drei willkürlich ausgewählten national führenden soziologischen Zeitschriften soll das gezeigt werden. Die drei Zeitschriften rangieren im SSCI unterschiedlich genug, um Generalisierungen zu erlauben. Die American Sociological Review ist in dem zwölfjährigen, weiter oben schon einmal herangezogenen Beobachtungszeitraum die Nummer 1, das British Journal of Sociology nimmt Rang 13 ein, und die Kölner Zeitschrift für Soziologie und Sozialpsychologie befindet sich auf Rang 67 (von 148 Zeitschriften).

In jedem Jahr entfällt bei jeder der drei Zeitschriften von der Gesamtzahl der Zitationen eine deutlich kleinere Zahl auf das unmittelbar davorliegende Jahr (und man kann dieses Muster mit einigem Recht auf die anderen soziologischen Zeitschriften verallgemeinern). Nehmen wir als Beispiel die ASR des Jahres 2008. Der offizielle Impact Faktor dividiert die Summe der Zahl der Zitationen, die auf Artikel entfallen, die in den beiden davorliegenden Jahren 2007 und 2006 (86 und 230) erschienen sind, durch die Zahl der Artikel, die im ASR in diesen beiden Jahren erschienen sind (42 und 42), woraus sich der Impact Faktor von 3,762 errechnet. Geht man nun bloß ein Jahr weiter nach hinten, ergibt sich eine Relation, die das Gesamtbild der Zitationen weitaus zutreffender wiedergibt: $230+252 / 42+41=$ 5,807. Ergebnis der kleinen Verschiebung des Beobachtungszeitraums ist, dass die ASR durchgehend einen deutlich höheren Impact Faktor aufweist, derjenige des BJS würde stetig ein wenig höher ausfallen, während die KZfSS nur geringfügig besser dastünde, was vermutlich das Ergebnis ihrer durchgehend verhältnismäßig geringen Zitationszahlen ist (und was seinerseits mit der Auswahl der im WoS aufgenommenen Zeitschriften zu tun hat: Jenseits der Grenzen des deutschen Sprachraums werden Artikel der KZfSS kaum zitiert). ${ }^{71}$ Wegen des völlig induktiven Vorgehens jener, die den ursprünglichen Impact Faktor kreierten, schiene mir eine Adaptierung, die der Gesamtverteilung der Daten Rechnung trägt, nicht nur erlaubt, sondern sogar geboten.

71 Dem korrespondiert der pragmatisch definierte Schwellenwert für den Zwei-JahresImpact Faktor, wonach Werte kleiner als 1,8 als wenig aussagekräftig betrachtet werden.

Leviathan, 41. Jg., 4/2013 
Tabelle 3: Zitationsverteilung für American Sociological Review, British Journal of Sociology und Kölner Zeitschrift für Soziologie und Sozialpsychologie

\begin{tabular}{|c|c|c|c|c|c|c|c|c|}
\hline & \multicolumn{8}{|c|}{ Jahr } \\
\hline & & 2011 & 2010 & 2009 & 2008 & 2007 & 2006 & 2005 \\
\hline \multicolumn{9}{|c|}{ Number of times articles published in \{year\}cited articles published in ASR } \\
\hline \multirow{7}{*}{ Citing journals } & 2005 & & & & & & & 18 \\
\hline & 2006 & & & & & & 24 & 96 \\
\hline & 2007 & & & & & 26 & 89 & 183 \\
\hline & 2008 & & & & 16 & 86 & 230 & 252 \\
\hline & 2009 & & & 22 & 109 & 168 & 262 & 317 \\
\hline & 2010 & & 15 & 143 & 182 & 258 & 329 & 332 \\
\hline & 2011 & 17 & 83 & 284 & 236 & 248 & 368 & 316 \\
\hline Number of items published in ASR in \{year\} & & & 39 & 44 & 44 & 42 & 42 & 41 \\
\hline »offizieller« 2-Jahres-IF & & 4,422 & 3,693 & 3,221 & 3,762 & 3,277 & 3,205 & 2,933 \\
\hline »offizieller« 2 Jahres-IF - 1 & & 5,909 & 5,116 & 5,119 & 5,807 & 4,551 & 4,747 & 4,329 \\
\hline \multicolumn{9}{|c|}{ Number of times articles published in $\{$ year $\}$ cited articles published in BJS } \\
\hline \multirow{7}{*}{ Citing journals } & 2005 & & & & & & & 16 \\
\hline & 2006 & & & & & & 28 & 13 \\
\hline & 2007 & & & & & 6 & 33 & 87 \\
\hline & 2008 & & & & 12 & 29 & 52 & 69 \\
\hline & 2009 & & & 21 & 24 & 73 & 94 & 64 \\
\hline & 2010 & & 74 & 34 & 72 & 82 & 111 & 76 \\
\hline & 2011 & 16 & 54 & 53 & 77 & 64 & 77 & 56 \\
\hline Number of items published in BJS in \{year\} & & & 39 & 27 & 30 & 27 & 28 & 21 \\
\hline »offizieller« 2-Jahres-IF & & 1,621 & 1,860 & 1,702 & 1,473 & 2,449 & 1,000 & 1,490 \\
\hline »offizieller« 2-Jahres-IF -1 & & 2,281 & 2,702 & 3,036 & 2,469 & 3,227 & 1,529 & 1,274 \\
\hline \multicolumn{9}{|c|}{ Number of times articles published in $\{$ year $\}$ cited articles published in KZfSS } \\
\hline \multirow{7}{*}{ Citing journals } & 2005 & & & & & & & 4 \\
\hline & 2006 & & & & & & 2 & 14 \\
\hline & 2007 & & & & & 4 & 19 & 11 \\
\hline & 2008 & & & & 1 & 23 & 34 & 15 \\
\hline & 2009 & & & 2 & 28 & 39 & 38 & 20 \\
\hline & 2010 & & 8 & 10 & 27 & 33 & 25 & 12 \\
\hline & 2011 & 1 & 29 & 31 & 40 & 21 & 41 & 24 \\
\hline Number of items published KZfSS in \{year\} & & & 70 & 47 & 22 & 24 & 24 & 25 \\
\hline "offizieller« 2-Jahres-IF & & 0,513 & 0,536 & 1,457 & 1,188 & 0,612 & 0,580 & 0,436 \\
\hline »offizieller« 2-Jahres-IF -1 & & 1,029 & 1,304 & 1,604 & 1,000 & 0,500 & 0,582 & 0,508 \\
\hline
\end{tabular}

Erläuterung: Um die Überprüfung zu erleichtern wurden die Originaltexte angeführt. Die Zeilen "Citing journals" geben die Gesamtsummen des jeweiligen Jahres an, im JCR findet man dort auch jede einzelne Zeitschrift angeführt.

Quellen: Cited journal data-Tabellen aus Journal Citation Report, verschiedene Jahrgänge, eigene Auszählung. 
ISIs Konkurrent Scopus berechnet seinen eigenen Einfluss-Faktor, ${ }^{72}$ doch ich verzichte hier darauf, diesen in analoger Weise zu überprüfen. Es mag genügen, darauf hinzuweisen, dass der Pearson-Korrelationskoeffizient für eine Stichprobe von 86 soziologischen Zeitschriften, die in beiden Datenbanken berücksichtigt sind, zwischen ISIs Fünf-Jahres-Impact Faktor und Scopus' SCImago Journal Rank (SJR) Faktor nur 0,582 beträgt (signifikant auf dem 0.01-Niveau). Für die numerische Feststellung von Qualitätsunterschieden zwischen Mengen von Aufsätzen, die in unterschiedlichen wissenschaftlichen Zeitschriften versammelt sind, eignen sich derartige Maßzahlen so lange nicht, so lange sie sich mit einer willkürlich verkleinerten Stichprobe der zitierten Literatur begnügen. In informeller Kommunikation über Zitationsindizes wird häufig auf Zitierkartelle als wichtigsten Faktor für Verzerrungen hingewiesen, doch diese an Verschwörungstheorien gemahnende Kritik scheint mir im Vergleich zu den technisch-rechnerischen Mängeln des Impact Faktors geradezu belanglos. Das Reaktivitätsphänomen soll damit aber nicht in Abrede gestellt werden, sondern bloß auf einen Platz in den hinteren Rängen verwiesen werden.

\section{Reliabilitätsprobleme}

Wenn man die Angaben des JCR für einzelne Zeitschriften genauer inspiziert, stößt man beispielsweise auf fehlende Fünf-Jahres-Impact Faktoren, kann diese aber aus den Daten, die für jede einzelne Zeitschrift angeführt werden, selbst errechnen. Gelegentlich kommen dabei überraschende Ergebnisse zum Vorschein: Der FünfJahres-Impact Faktor des Journal of Consumer Culture übertrifft beispielsweise mit 7,13 alle prominenten amerikanischen Zeitschriften bei Weitem, und das European Journal of Social Theory liegt mit seinen 3,33 noch vor dem BJS. Doch warum unterlässt Thomson Reuters deren Bekanntmachung? Die naheliegende Antwort, das geschehe, weil die Daten für die Zitationen oder die Zahl der veröffentlichten Artikel fehlen, stimmt nicht, da bei anderen Zeitschriften, wie beispielsweise Eastern European Countryside, Current Perspectives in Social Theory, mit vergleichbaren Datenlöchern deren Fünf-Jahres-Impact Faktoren dennoch berichtet werden. Viel wahrscheinlicher unterließ Thomson Reuters die Bekanntgabe des bemerkenswert hohen Fünf-Jahres-Impact Faktor des Journal of Consumer Culture, weil es den Erwartungen der Nutzer widersprechen würde, dass ein vergleichsweise randständiges Journal die etablierten $A S R, A J S$ etc. übertrifft. Doch kann man Thomson Reuters Informationspolitik dann überhaupt noch vertrauen?

Ich verglich die Daten des JCR für verschiedene Zeitschriften mit jenen aus dem SSCI und fand heraus, dass die Angaben des JCR im SSCI nicht reproduziert werden

72 González-Pereira et al. o. J.

Leviathan, 41. Jg., 4/2013 
konnten. ${ }^{73}$ Die Analysen ergaben vielfach Nichtübereinstimmungen, die sich vor allem beim Versuch ergaben, das Bezugsjahr (»timespan«) zu modellieren: Die »cites in [year] « lassen sich im SSCI nicht exakt reproduzieren. ${ }^{74}$

Ein genauerer Blick auf die Daten hinter den summarischen Berichten des JCR führte zu weiteren aufschlussreichen Entdeckungen. Am Beispiel der russischen Zeitschrift Sotsiologicheskie Issledovaniya (übersetzt in etwa: Soziologische Studien), die im SSCI seit mindestens 2000 erfasst wird, soll das stellvertretend gezeigt werden. Der Impact Faktor dieser Zeitschrift wurde für 2010 mit 0,147 angegeben und der Fünf-Jahres-Impact Faktor mit 0,135 (der von mir berechnete Mittelwert des zweijährigen Impact Faktors über zwölf Jahre hinweg ist 0,144). Damit lag diese Zeitschrift auf den Rängen 119 (für den zweijährigen Impact Faktor) beziehungsweise 93 (für den Fünf-Jahres-Impact Faktor). Im Jahr 2010 wurden »articles « und »reviews ", die in dieser Zeitschrift in den beiden Jahren davor 2008 und 2009 erschienen sind, 64-mal zitiert; im selben Zweijahres-Zeitraum erschienen in dieser Zeitschrift zusammen bemerkenswerte 436 »articles « und »reviews «. 64 dividiert durch 436 ergibt 0,147. Alles scheint in Ordnung. Doch wenn man auf der JCRSeite ein wenig nach unten blättert, findet man einen Link, der einen zur »cited journal data table « führt, wo die "number of times articles published in 2010 (in journals below) cited articles published in SOTSIOL ISSLED+ [das ist das von ISI verwendete Akronym für diese Zeitschrift] (in years below) « findet. Dort sieht man dann, dass 47 der 64 Zitierungen in eben dieser Zeitschrift erschienen. Eine SelbstZitationsrate von 73 Prozent ist jedenfalls bemerkenswert. In den Anfangsjahren des Zitationsbetriebs schlugen manche vor, überhaupt alle Selbstzitate aus den Berechnungen auszuschließen (was vermutlich verworfen wurde, weil es zeitaufwändig gewesen wäre). Später einigte man sich auf einen Grenzwert von einem Fünftel, weil man herausgefunden hatte, dass nur rund 18 Prozent aller Zeitschriften diesen Wert an Selbstzitaten übertrafen. ${ }^{75}$

Es wäre wohl nicht unbillig, zu erwarten, dass Thomson Reuters Zeitschriften, die über längere Zeiträume hinweg vor allem durch Selbstzitationen auffallen, aus

73 Ich bin keineswegs der erste, der auf derartige Inkonsistenzen hinweist. In Scientometrics, einer spezialisierten Zeitschrift, die im SSCI im subject »Information science \& library science (mit einem Impact Faktor von 1,9) geführt wird, gibt es eine Menge kritischer Beiträge, doch finden sie jenseits der engen Zirkel der Bibliometriker selten Gehör. Siehe zum Beispiel: Glänzel, Moed 2002; Archambault, Larivière 2009. Ein dort kürzlich veröffentlichter kritischer Beitrag löste eine bemerkenswerte Zahl an Repliken aus (Vanclay 2012). Am aufschlussreichsten sind: Ingwersen 2012; Moed et al. 2012; Pudovkin, Garfield 2012; Smith 2012; Zitt 2012.

74 Ich führte im Mai 2012 folgende Suche durch: »Cited Work=([journal title]) AND Cited Year $=(2005-2009)$. Refined by: Document Type $=($ ARTICLE or REVIEW $)$. Databases=SSCI. Timespan=2000-2010 « und wiederholte danach die Suche für »cited year=(2008-2009) «. Damals gelang es nicht, die JCR-Daten zu rekonstruieren. Einige Monate später konnte ich diese Suche nicht replizieren, da das WoS die Suchoptionen geändert hatte (der »timespan " muss nun anders definiert werden). Es war daher nicht möglich, die erste Suche in befriedigender Weise zu replizieren.

75 Vanclay fasst die Debatten in seiner Table 2 zusammen (Vanclay 2012). 
der Datenbank eliminiert oder zumindest - analog zu dem oben erwähnten zu hohen Fünf-Jahres-Impact Faktor des Journal of Consumer Culture - solche Werte in den entsprechenden Jahren nicht ausweist. Dem Tun auf den hinteren Rängen des WoS schenkt Thomson Reuters offenbar jedoch nur wegen der Käufer Beachtung, nicht aber um der Integrität des Impact Faktors wegen.

\section{Folgerungen}

Die wesentlichen Befunde dieser Studie zusammenfassend, würde ich die Aufmerksamkeit gerne auf folgende Schwächen des Zitations-Index-Geschäfts und seines Leuchtturms Impact Faktor lenken. Sie können in zwei Gruppen geteilt werden: einerseits intrinsische Defizite der Prozeduren und anderseits spezifische Mängel im Bereich der Sozialwissenschaften.

(1) Der Impact Faktor wurde unter Heranziehung von Daten aus den Naturwissenschaften induktiv entwickelt. Dabei wurde nie eine theoretisch befriedigende Begründung für die sich dann durchsetzende Formel geboten. Deren Verbreitung war vielmehr der Nebeneffekt der hartnäckigen Verwendung einer anfangs plausibel erscheinenden Maßzahl, die nach einiger Zeit nahezu von allen als valide Messung akzeptiert wurde. Der in diesem Feld dominante Induktivismus wird auch daran ersichtlich, dass ständig neue Indikatoren ausgearbeitet und vorgeschlagen werden. ${ }^{76}$ Insbesondere das zweijährige Zitationsfenster ist völlig willkürlich, da es bestenfalls in gut integrierten Forschungsfeldern mit einem hohen Grad an Konsens darüber, was als nächstes erforscht werden soll, anwendbar ist.

(2) Die Kriterien, was als zitierbares Dokument betrachtet wird und welche Textsorten als Quellen von Zitationen berücksichtigt werden, sind alles andere als gut definiert. Die Nichtübereinstimmung der Definitionen für Zähler beziehungsweise Nenner des Impact Faktors - eine größere Zahl oberhalb des Trennstrichs und eine kleinere unterhalb - wirkt geradezu als Einladung zur Manipulation, etwa durch die Veröffentlichung von Editorials (eine im Nenner nicht zu berücksichtigende Textsorte) mit einer Menge von Selbstzitaten (die in den Zähler eingehen).

(3) Die Messung ist hochgradig reaktiv und wird das immer mehr, da Autoren, Herausgeber, Verleger und andere interessierte Parteien ihr eigenes Verhalten den Messgewohnheiten anpassen können. Darunter leidet die Objektivität, weil Zitierkartelle, die Zerlegung von Texten in die kleinsten noch publizierbaren Einheiten, Ehrenautorenschaft und verwandte soziale Technologien des wissenschaftlichen Arbeitens mittlerweile selbst jenen bekannt sind, die niemals das Feld wissenschaftlichen Veröffentlichens betreten werden. Ethikkodizes und

76 Vgl. als neueren Überblick Pendlebury 2009. Dem Outsider erscheinen manche Beiträge gelegentlich wie unfreiwillige Parodien auf die Arbeit von Wissenschaftlern. Man konsultiere dazu beispielsweise die »Proceedings of 17th International Conference on Science and Technology Indicators" (Archambault et al. 2012) und lese den Beitrag der beiden führenden Szientometriker Glänzel und Moed (2012). 
vergleichbare Maßnahmen bestätigen, dass deviantes Verhalten existiert, aber dessen Ausmaß ist unbekannt und umstritten. ${ }^{77}$

(4) Das WoS wurde ursprünglich in den USA entwickelt, und die Erfassung der nichtamerikanischen Wissenschaftswelt ist bis heute unvollständig geblieben, was sich vor allem in Disziplinen auswirkt, in denen eine wohldefinierte kosmopolitische Forschungsagenda fehlt. Die Auswahl der zu berücksichtigenden Zeitschriften ist zumindest undurchsichtig. Sogar der Gründer von ISI, Eugene Garfield, gesteht ein, dass es sich um eine Mischung dessen handelt, was er "quantitative and qualitative Indikatoren « nennt:

"Zitationsdaten sind eine Quelle von quantitativen Indikatoren, die zur Auswertung
von Zeitschriften mit etablierter Erfolgsgeschichte benutzt werden können. Aber die
Auswahl von Zeitschriften beruht oft auf anderen, eher qualitativen Erwägungen. Zeit-
schriftenstandards sind ein Beispiel. Dass eine Zeitschrift stets ihre Erscheinungstermine
einhalten kann, ist vielleicht die grundlegendste Erwartung. [...] Editorische Anforde-
rungen an Zusammenfassungen, Titel und Referenzen [...] Begutachtung eingereichter
Manuskripte, die Mitgliedschaft im Herausgebergremium und die Reputation des Ver-
lags [...] sind weitere Indikatoren für die Qualität einer Zeitschrift «. ${ }^{8}$

Nach dem Verkauf von ISI an Thomson Reuters wurden die Auswahlverfahren zunehmend stärker von Faktoren bestimmt, die mit der Feststellung der wissenschaftlichen Verdienste der Berücksichtigten, also ihrer Reputation, weniger zu tun hatten.

Im Fall der Sozialwissenschaften lassen einige ihrer Besonderheiten die Angemessenheit der Routinen des WoS fraglich erscheinen.

(1) Die Gliederung und Untergliederung des gesamten Felds ist nicht nachvollziehbar. Disziplinen von vergleichbarer Größe und ähnlich gelagertem Publikationsverhalten, wie Psychologie und Ökonomie (zwei mittlerweile geradezu paradigmatische Zeitschriften-Wissenschaften), sind extrem ungleich vertreten. Niemandem kann glauben gemacht werden, dass die Psychologie doppelt so groß (oder: bedeutsam) ist wie die Ökonomie, und in gleicher Weise ist zu bezweifeln, dass die Soziologie nur halb so groß wie die Psychologie sei. Die Ausweitung des Felds der Sozialwissenschaften erfolgte nicht nach wissenschaftlichen Kriterien: Sowohl die Einbeziehung einiger Felder angewandter Forschung wie die Einbeziehung befremdlicher Spezialgebiete wie »ergonomics «, »substance abuse « und »transport « führten dazu, dass die Grenzen des Gebiets, aus dem Zitationen kommen können, in willkürlicher Weise erweitert wurden.

(2) Angesichts des niedrigen Niveaus an internationalem Konsens über die Forschungsagenda in den verschiedenen Teilen der Sozialwissenschaften, was auch als Fragmentierung bezeichnet wird, ist jede Auswahl von Zeitschriften schwierig und zugleich umstritten. Tatsächlich folgt das WoS keinem der dort zu irgendeinem Zeitpunkt kundgemachten Kriterien, sondern den Markterobe-

77 Jüngst machte eine Studie auf das beunruhigende Ausmaß erzwungener Zitierungen aufmerksam: Wilhite, Fong 2012 sowie ergänzendes Online-Material: www.sciencemag.org/cgi/content/full/335/6068/542/DC1 (Zugriff vom 14.06.2013).

78 Garfield 1990, S. 185. 
rungsstrategien ihres heutigen Eigentümers, dem internationalen Informations-, Wissenschafts- und Medienkonzern Thomson Reuters. Gemäß den eigenen Standards dürfte die überwiegende Zahl der im SSCI erfassten Zeitschriften wegen mangelnder Reputation eigentlich nicht Berücksichtigung finden. ${ }^{79}$

(3) Die wissenschaftlichen Gewohnheiten der Soziologen und vermutlich auch diejenigen anderer Sozialwissenschaftler finden keine Entsprechung in der willkürlichen Festlegung des Zitationsfensters von nur zwei Jahren. Auch schon eine kursorische Inspektion der Literaturverzeichnisse von Abhandlungen beliebiger soziologischer Zeitschriften offenbart, dass sich der Großteil der Verweise auf Titel bezieht, die älter als zwei Jahre sind. Ein ohnehin bloß induktiv etabliertes Kriterium, das den Praktiken der Mehrzahl der nicht-naturwissenschaftlichen Disziplinen widerspricht, sollte daher eigentlich nicht verwendet werden.

(4) Der oftmals beklagte "methodologische Nationalismus « 80 ist eine weitere Besonderheit der Sozialwissenschaften und insbesondere der Soziologie, der zur Folge hat, dass Veröffentlichungen von Soziologen aus kleineren Staaten notwendigerweise weniger Anerkennung im Wege von Zitationen erzielen können. Selbst jene Zeitschriften, die die englische Sprache benutzen, können nationalkulturell etablierte Beschränkungen des Wahrnehmungshorizonts nicht überwinden. Analoges gilt vermutlich auch für all jene spezialisierten Forschungsfelder, die nur von einer vergleichsweise kleinen Zahl von Wissenschaftlern bearbeitet werden. Je größer die Zahl der an einem Feld Beteiligten, desto höher die Zahl der veröffentlichten Aufsätze und damit auch die Summe der Zitationen.

Einige Tage des Datenschaufelns legen den Schluss nahe, dass es sich bei dem Getue um den Impact Faktor letztlich nur um etwas handelt, was Karl Marx schon vor Langem den Mechanismus des Warenfetischismus genannt hat:

»Es ist nur das bestimmte gesellschaftliche Verhältnis der Menschen selbst, welches hier für sie die phantasmagorische Form eines Verhältnisses von Dingen annimmt. Um daher eine Analogie zu finden, müssen wir in die Nebelregion der religiösen Welt flüchten. Hier scheinen die Produkte des menschlichen Kopfes mit eignem Leben begabte, untereinander und mit den Menschen in Verhältnis stehende selbständige Gestalten. So in der Warenwelt die Produkte der menschlichen Hand. Dies nenne ich den Fetischismus, der den Arbeitsprodukten anklebt, sobald sie als Waren produziert werden, und der daher von der Warenproduktion unzertrennlich ist «. ${ }^{81}$

Das jüngst etablierte Regime des Impact Faktor wird durch die Verkaufsinteressen zweier internationaler Wissenschaftskonzerne, Thomson Reuters und Elsevier,

79 Zwei von drei Zeitschriften der Ökonomie, 42 Prozent der »family studies «, jeweils 38 Prozent in Soziologie und »substance abuse « sowie 35 Prozent über alle Teilbereiche der Psychologie hinweg weisen einen Impact Faktor kleiner als 1,0 auf; alle diese werden aber übertroffen von den Zeitschriften der Geschichtswissenschaft, von denen nur eine einen Impact Faktor größer als 1,0 hat, 97 Prozent aber darunter liegen.

80 Dieser Begriff wurde, obwohl er im Deutschen mit Ulrich Beck assoziiert zu werden pflegt, zuerst systematisch von Martins 1974 und Smith 1983 eingeführt. Vgl. Wimmer, Glick Schiller 2003.

81 Marx 1968 [1867], S. 86 f.

Leviathan, 41. Jg., 4/2013 
angetrieben und wurde in der akademischen Welt von der immer bedeutender werdenden neuen Elite der Universitäts- und Wissenschaftsadministratoren zum GoldStandard erhoben, dem sich merkwürdigerweise auch Wissenschaftler immer dann unterordnen, wenn derartige Kennzahlen ihrem Eindrucksmanagement dienlich sind. Charles Goodharts (von anderen zu einem nach ihm benannten »Gesetz « erhobene) Beobachtung, die diesem Artikel als Motto vorangestellt wurde, trifft auf den Fall des Impact Faktors jedoch nicht zu, da jene, die den Impact Faktor kritisieren, das bislang eher in informeller Kommunikation taten oder ihre Einwände meist nur innerhalb des Spezialistendiskurses der Szientometrie publik machten und daher weder beim breiteren Fach- noch beim allgemeinen Publikum Gehör fanden. Maße wie der Impact Faktor, die von Beginn an keine guten war, können durch eine Diskussion über sie allerdings auch nicht besser werden. Als Korollar zu Goodharts Gesetz könnte man dann formulieren, dass ein Maß, das immer schon schlecht war, dennoch einer Diskussion bedarf, um seinen dürftigen Charakter vorzuführen.

\section{Literatur}

Aaltojärvi, Inari et al. 2008. »Scientific productivity, web visibility and citation patterns in sixteen nordic sociology departments ", in Acta Sociologica 51, 1, S. 5-22.

Abbott, Andrew 2011. »Library research infrastructure for humanistic and social scientific scholarship in America in the twentieth century ", in Social knowledge in the making, hrsg. v. Camic, Charles; Lamont, Michèle; Gross, Neil, S. 43-87. Chicago: University of Chicago Press.

Archambault, Éric; Gingras, Yves; Larivière, Vincent. Hrsg. 2012. Proceedings of 17th international conference on science and technology indicators. 2 Bände. Montréal: Science-Metrix and OST.

Archambault, Éric; Larivière, Vincent 2009. »History of the Journal Impact Factor: contingencies and consequences", in Scientometrics 79, 3, S. 635-649.

Ashton, Susan V.; Oppenheim, Charles 1978. "A method of predicting Nobel prizewinners in chemistry ", in Social Studies of Science 8, 3, S. 341-348.

Baldi, Stéphane 1998. "Normative versus social constructivist processes in the allocation of citations: a network-analytic model ", in American Sociological Review 63, 6, S. 829-846.

Baum, Joel A. C. 2011. »Free-riding on power laws: questioning the validity of the Impact Factor as a measure of research quality in organization studies ", in Organization 18, 4, S. 449-466.

Becker, Joseph 1968. »Information storage and retrieval ", in International encyclopedia of the social sciences, Band 7, hrsg. v. Sills, David L., S. 301-304. New York: Macmillan.

Bensman, Stephen J. 2007. "Garfield and the Impact Factor ", in Annual Review of Information Science and Technology 41, 1, S. 93-155.

Bjarnason, Thoroddur; Sigfusdottir, Inga Dora 2002. "Nordic impact: article productivity and citation patterns in sixteen Nordic sociology departments", in Acta Sociologica 45, 4, S. 253-267.

Bulmer, Martin; Solomos, John 2010. »Introduction: developments and plans for the future of ethnic and racial studies ", in Ethnic and Racial Studies 33, 10, S. 1689.

Chi, Pei-Shan 2012. "Characteristics of publications in political science ", in Proceedings of 17th international conference on science and technology indicators, 2 Bände, hrsg. v. Archambault, Éric; Gingras, Yves; Larivière, Vincent, S. 441-442. Montréal: Science-Metrix and OST.

Collini, Stefan 2012. What are universities for? London: Penguin.

Drori, Gili S. et al. 2003. Science in the modern world polity. Institutionalization and globalization. Stanford: Stanford University Press.

Elkana, Yehuda et al. Hrsg. 1978. Toward a metric of science. The advent of science indicators. New York: Wiley. 
Engels, Tim; Ossenblok, Truyken; Spruyt, Eric 2012. »Changing publication patterns in the social sciences and humanities, 2000-2009", in Scientometrics 93, 2, S. 373-390.

Europa Publications 2011. The Europa world of learning 2012. 62nd edition. London: Routledge.

European Commission 2011. The ideas work programme. European Research Council work programme 2012. Brüssel: European Commission.

Fabel, Oliver 2008. "Editorial: Zum Schwerpunkt Forschungs-Rankings ", in Perspektiven der Wirtschaftspolitik 9, 3, S. 252-253.

Fischer, Klaus 2008. "Science and its malfunctions", in Human Architecture: Journal of the Sociology of Self-knowledge 6, 2, S. 1-22.

Fleck, Christian 2010. »Wie statistische und andere Zahlen unser Vertrauen gewinnen und enttäuschen ", in Wabrheit ... Herstellung, Nutzen und Gebrauch von » Wabrheit " in Wissenschaft und Alltag, hrsg. v. Aulenbacher, Brigitte; Ziegler, Meinrad, S. 131-146. Innsbruck: Studienverlag (transblick).

Fox, Robert et al. 2009. "Journals under threat: a joint response from history of science, technology and medicine editors", in Notes and Records of the Royal Society 63, S. 1-3.

Fuyuno, Ichiko; Cyranoski, David 2006. "Cash for papers: putting a premium on publication ", in Nature 441, S. 792.

Garfield, Eugene 1963. "Citation indexes in sociological and historical research «, in American Documentation 14, 4, S. 289-291.

Garfield, Eugene 1971. »The mystery of transposed journals lists - wherein Bradford's law of scattering is generalized according to Garfield's law of concentration «, in Current Contents 17, S. 5-6.

Garfield, Eugene 1972. "Citation analysis as a tool in journal evaluation ", in Science Citation Index 1961 178, S. 471-479.

Garfield, Eugene 1973. »More on forecasting Nobel Prizes and the most cited scientists of 1972 «, in Current Contents 40, S. 5-7.

Garfield, Eugene 1977 a. »Citation analysis as a tool in journal evaluation «, in Eugene Garfield: Essays of an information scientist: 1962-1973, S. 527-544. Philadelphia: ISI-Press (1).

Garfield, Eugene 1977 b. »Citation indexing, historio-bibliography, and the sociology of science. [1970]", in Eugene Garfield: Essays of an information scientist: 1962-1973, S. 158-174. Philadelphia: ISI-Press (1).

Garfield, Eugene 1977 c. » (Citations-to< divided by >items-published ‘ gives Journal Impact Factor. ISI lists the top fifty high-impact journals of science", in Eugene Garfield: Essays of an Information Scientist: 1962-1973, S. 270-273. Philadelphia: ISI-Press (1).

Garfield, Eugene 1988. »Derek Price and the practical world of scientometrics «, in Science, Technology, \& Human Values 13, 3/4, S. 349-350.

Garfield, Eugene 1990. »How ISI selects journals for coverage: quantitative and qualitative considerations ", in Current Contents 22, S. 5-13.

Garfield, Eugene 2005. The agony and the ecstacy - the history and meaning of the Journal Impact Factor. International congress on peer review and biomedical publication. Chicago, 16. September 2005. http://garfield.library.upenn.edu/papers/jifchicago2005.pdf (Zugriff vom 14.06.2013).

Garfield, Eugene; Sher, Irving H. 1963. »New factors in the evaluation of scientific literature through citation indexing «, in American Documentation 14, 3, S. 195-201.

Gingras, Yves; Mosbah-Natanson, Sébastien 2010. "Where are social sciences produced? ", in World social science report 2010. Knowledge divides, hrsg. v. UNESCO; ISSC, S. 149-153. Paris: UNESCO Publishing.

Glänzel, Wolfgang; Moed, Henk 2002. »Journal impact measures in bibliometric research «, in Scientometrics 53, 2, S. 171-193.

Glänzel, Wolfgang; Moed, Henk F. 2012. "Thoughts and facts on bibliometric Indicators «, in Proceedings of 17th international conference on science and technology indicators, 2 Bände, hrsg. v. Archambault, Éric; Gingras, Yves; Larivière, Vincent, S. 305-318. Montréal: ScienceMetrix and OST.

González-Pereira, Borja; Guerrero-Bote, Vicente; Moya-Anegón, Felix o. J. The SJR indicator: a new indicator of journals' scientific prestige. http://arxiv.org/ftp/arxiv/papers/ 0912/0912.4141.pdf (Zugriff vom 14.06.2013).

Leviathan, 41. Jg., 4/2013 
Hargens, Lowell L. 2000. »Using the literature: reference networks, reference contexts, and the social structure of scholarship", in American Sociological Review 65, 6, S. 846-865.

Holmwood, John 2010. "Sociology's misfortune: disciplines, interdisciplinarity and the impact of audit culture ", in British Journal of Sociology 61, 4, S. 639-658.

Ingwersen, Peter 2012. "The pragmatics of a diachronic Journal Impact Factor", in Scientometrics 92, 2, S. 319-324.

Kaplan, Norman; Storer, Norman W. 1968. "Scientific communication", in International encyclopedia of the social sciences, Vol. 14, hrsg. v. Sills, David L., S. 112-117. New York: Macmillan.

Kieser, Alfred 2010. »Unternehmen Wissenschaft? «, in Leviathan 38, 3, S. 347-367.

Kieser, Alfred 2012. "JOURQUAL. Der Gebrauch, nicht der Missbrauch, ist das Problem. Oder: warum Wirtschaftsinformatik die beste deutschsprachige betriebswirtschaftliche Zeitschrift ist ", in Die Betriebswirtschaft: DBW 72, 1, S. 93-110.

Kirchner, Frank. Hrsg. 2004. World guide to scientific associations and learned societies. Handbook of international documentation and information 13. 9. Auflage. München: Saur.

Lederberg, Joshua 2000. »How the science citation index got started «, in The web of knowledge: a Festschrift in honor of Eugene Garfield, hrsg. v. Cronin, Blaise; Barsky Atkins, Helen, S. 25-64. Mulford: Information Today Inc.

Macdonald, Stuart; Kam, Jacqueline 2007. "Ring a ring o' roses: quality journals and gamesmanship in management studies ", in Journal of Management Studies 44, 4, S. 640-655.

Macdonald, Stuart; Kam, Jacqueline 2011. »The skewed few: people and papers of quality in management studies ", in Organization 18, 4, S. 467-475.

Martins, H. 1974. "Time and theory in sociology «, in Approaches to sociology. An introduction to major trends in British sociology, hrsg. v. Rex, John, S. 246-294. London: Routledge \& Kegan Paul.

Martyn, John; Gilchrist, Alan 1968. An evaluation of British scientific journals. Aslib. Occasional Publication 1. London: Aslib.

Marx, Karl 1968 [1867]. Das Kapital. Band 1: Kritik der politischen Ökonomie. Marx-EngelsWerke, Band 23. Berlin: Dietz.

Merton, Robert K. 1979 a. "Foreword ", in Eugene Garfield: Citation indexing. Its theory and application in science, technology, and humanities, S. v-ix. New York: John Wiley \& Sons.

Merton, Robert K. 1979 b. »The evolving grammar of citation analysis ", in Eugene Garfield: Citation indexing: its theory and application in science, technology and humanities, S. vii-xi. New York: John Wiley \& Sons.

Merton, Robert K. 2000. "On the Garfield input to the sociology of science: a retrospective collage ", in The web of knowledge: a Festschrift in honor of Eugene Garfield, hrsg. v. Cronin, Blaise; Barsky Atkins, Helen, S. 435-448. Mulford: Information Today Inc.

Merton, Robert K.; Garfield, Eugene 1986. "Foreword «, in Little science, big science... and beyond, hrsg. v. Price, Derek Solla de, S. vii-xii. New York: Columbia University Press.

Meyer, John W.; Rowan, Brian 1977. »Institutionalized organizations: formal structure as myth and ceremony ", in American Journal of Sociology 83, 2, S. 340-363.

Mirowski, Philip 2011. Science-mart. Privatizing American science. Cambridge: Harvard University Press.

Moed, Henk et al. 2012. "Citation-based metrics are appropriate tools in journal assessment provided that they are accurate and used in an informed way ", in Scientometrics 92, 2, S. 367-376.

Münch, Richard 2007. Die akademische Elite. Zur sozialen Konstruktion wissenschaftlicher Exzellenz. Frankfurt a. M.: Suhrkamp.

Pendlebury, David A. 2009. "The use and misuse of journal metrics and other citation indicators", in Archivum Immunologiae et Therapiae Experimentalis 57, 1, S. 1-11.

Persson, Olle 1985. "Scandinavian social science in international journals", in Social Science Information Studies 5, 4, S. 185-190.

Power, Michael 1997. The audit society. Rituals of verification. New York: Oxford University Press.

Price, Derek Solla de 1978. »Editorial statements«, in Scientometrics 1, S. 7. 
Pudovkin, Alexander I.; Garfield, Eugene 2012. »Rank normalization of Impact Factors will resolve Vanclay's dilemma with TRIF. Comments on the paper by Jerome Vanclay ", in Scientometrics 92, 2, S. 409-412.

Sauder, Michael; Espeland, Wendy Nelson 2009. »The discipline of rankings: tight coupling and organizational change", in American Sociological Review 74, 1, S. 63-82.

Schneider, Friedrich 2008. "Special issue on the economics of the economics profession. Editorial ", in German Economic Review 9, 4, S. 397-401.

Schofer, Evan 2004. »Cross-national differences in the expansion of science, 1970-1990 «, in Social Forces 83, 1, S. 215-248.

Sica, Alan 2012. "Editor's remarks «, in Contemporary Sociology 41, 2, S. 137-138.

Smith, Anthony D. 1983. "Nationalism and classical social theory «, in British Journal of Socio$\log y 34,1$, S. 19-38.

Smith, Derek R. 2012. »Impact factors, scientometrics and the history of citation-based research «, in Scientometrics 92, 2, S. 419-427.

Strathern, Marilyn 2000. Audit cultures. Anthropological studies in accountability, ethics, and the academy. London: Routledge.

Streeck, Wolfgang; Feick, Jürgen 2012. »Impact Factor and SASE's annual prize«, in SocioEconomic Review 10, 4, S. 625.

Testa, Jim 2012. The Thomson Reuters journal selection process. http://thomsonreuters.com/ products_services/science/free/essays/journal_selection_process/(Zugriff vom 14.06.2013).

UNESCO; ISSC. Hrsg. 2010. World social science report 2010. Knowledge divides. Paris: UNESCO Publishing.

Vanclay, Jerome 2012. »Impact Factor: outdated artefact or stepping-stone to journal certification? «, in Scientometrics 92, 2, S. 211-228.

Ward, Kevin 2010. »Research monographs: an overview ", in World social science report 2010. Knowledge divides, hrsg. v. UNESCO; ISSC, S. 300-302. Paris: UNESCO Publishing.

Wilhite, Allen W.; Fong, Eric A. 2012. »Coercive citation in academic publishing «, in Science 335, S. 542-543.

Willmott, Hugh 2011. »Journal list fetishism and the perversion of scholarship: reactivity and the ABS list «, in Organization 18, 4, S. 429-442.

Wimmer, Andreas; Glick Schiller, Nina 2003. »Methodological nationalism, the social sciences, and the study of migration. An essay in historical epistemology «, in International Migration Review 37, 3, S. 576-610.

Wouters, Paul; Leydesdorff, Loet 1994. »Has Price's dream come true: is scientometrics a hard science?", in Scientometrics 31, 2, S. 193-222.

Zitt, Michel 2012. »The Journal Impact Factor: angel, devil, or scapegoat? A comment on J.K. Vanclay's article 2011", in Scientometrics 92, 2, S. 485-503. 
Zusammenfassung: Der Beitrag untersucht Entstehung und Verwendung des Impact Faktors. Er war ein Nebenprodukt von Eugene Garfields Experimentieren mit den riesigen Datenmengen, die seinem Institute for Scientific Information zur Verfügung standen, um den Erfolg (impact) von Artikeln und Zeitschriften zu messen. Gänzlich induktiv wurde er durch eingehende Überprüfung der Daten und nach Konsultationen mit Experten aus verschiedenen naturwissenschaftlichen Disziplinen schrittweise entwickelt und danach ohne weitere Prüfung auf andere Disziplinen übertragen. Weder die Definition der Disziplinen noch die Auswahl der Zeitschriften, die in das Web of Science beziehungsweise den Social Science Citation Index aufgenommen wurden, sind nachvollziehbar begründet. Trotz offensichtlicher Ungeeignetheit wird der Impact Faktor nicht nur von Verlagen, sondern auch von Herausgebern sozialwissenschaftlicher Zeitschriften für das Eindrucksmanagement benutzt. Eine Form von Fetischismus scheint die Soziologen, die es besser wissen sollten, zu beherrschen.

Stichworte: Impact Faktor, Web of Science/Social Science Citation Index, Eugene Garfield, wissenschaftliche Zeitschriften, europäische Soziologie, Messen, Szientometrie

\section{The fetishism of the impact factor}

Summary: Scientometric measurement became widely used in recent times. One of the most popular indicators is the Impact Factor. This paper examines the coming into being of this highly influential figure. It is the offspring of Eugene Garfield's experimentation with the huge amounts of data available at his Institute for Scientific Information and the result of a number of attempts to find appropriate measurements for the success (»impact «) of articles and journals. The completely inductive procedure was initially adjusted by examining the data thoughtfully and by consultating with experts from different science disciplines. Afterwards, its calculation modes have been imposed on other disciplines without further consideration. The paper demonstrates in detail the inopportune consequences in particular for sociology. Neither the definition of disciplines, nor the selection of journals for the Web of Science/Social Science Citation Index follows any comprehensible rationale. The procedures for calculating the impact factor are inappropriate and suffer from a lack of similar inductive adjustments. Despite its obvious unsuitability, the impact factor is used by editors of sociological journals for marketing and impression management purposes. Fetishism seems to be ruling sociologists who should know better.

Keywords: Impact Factor, Web of Science/Social Science Citation Index, Eugene Garfield, journals, European sociology, measurement, scientometrics

Autor

Christian Fleck

Institut für Soziologie der Universität Graz

Universitätsstrasse 15

8010 Graz

Österreich

christian.fleck@uni-graz.at 